San Jose State University

SJSU ScholarWorks

Master's Theses

Master's Theses and Graduate Research

Fall 2010

\title{
Cooperative Symbol-Based Signaling for Networks with Multiple Relays
}

Yuanyuan Quan

San Jose State University

Follow this and additional works at: https://scholarworks.sjsu.edu/etd_theses

\section{Recommended Citation}

Quan, Yuanyuan, "Cooperative Symbol-Based Signaling for Networks with Multiple Relays" (2010).

Master's Theses. 3891.

DOI: https://doi.org/10.31979/etd.aypz-x2ra

https://scholarworks.sjsu.edu/etd_theses/3891

This Thesis is brought to you for free and open access by the Master's Theses and Graduate Research at SJSU ScholarWorks. It has been accepted for inclusion in Master's Theses by an authorized administrator of SJSU ScholarWorks. For more information, please contact scholarworks@sjsu.edu. 


\title{
COOPERATIVE SYMBOL-BASED SIGNALING FOR NETWORKS WITH MULTIPLE RELAYS
}

\author{
A Thesis \\ Presented to \\ The Faculty of the Department of Electrical Engineering \\ San José State University
}

\author{
In Partial Fulfillment \\ of the Requirements for the Degree \\ Masters of Science
}

by

Yuanyuan Quan

December 2010 
(C) 2010

Yuanyuan Quan

ALL RIGHTS RESERVED 
The Designated Thesis Committee Approves the Thesis Titled

COOPERATIVE SYMBOL-BASED SIGNALING FOR NETWORKS WITH

MULTIPLE RELAYS

by

Yuanyuan Quan

APPROVED FOR THE DEPARTMENT OF ELECTRICAL ENGINEERING

SAN JOSÉ STATE UNIVERSITY

December 2010

Dr. Birsen Sirkeci-Mergen

Department of Electrical Engineering

Dr. Essam Marouf

Department of Electrical Engineering

Dr. Robert H. Morelos-Zaragoza

Department of Electrical Engineering 


\section{ABSTRACT \\ COOPERATIVE SYMBOL-BASED SIGNALING FOR NETWORKS WITH MULTIPLE RELAYS \\ by Yuanyuan Quan}

Wireless channels suffer from severe inherent impairments and hence reliable and high data rate wireless transmission is particularly challenging to achieve. Fortunately, using multiple antennae improves performance in wireless transmission by providing space diversity, spatial multiplexing, and power gains. However, in wireless ad-hoc networks multiple antennae may not be acceptable due to limitations in size, cost, and hardware complexity. As a result, cooperative relaying strategies have attracted considerable attention because of their abilities to take advantage of multi-antenna by using multiple single-antenna relays.

This study is to explore cooperative signaling for different relay networks, such as multi-hop relay networks formed by multiple single-antenna relays and multi-stage relay networks formed by multiple relaying stages with each stage holding several single-antenna relays. The main contribution of this study is the development of a new relaying scheme for networks using symbol-level modulation, such as binary phase shift keying (BPSK) and quadrature phase shift keying (QPSK). We also analyze effects of this newly developed scheme when it is used with space-time coding in a multi-stage relay network. Simulation results demonstrate that the new scheme outperforms previously proposed schemes: amplify-and-forward (AF) scheme and decode-and-forward (DF) scheme. 


\section{ACKNOWLEDGEMENTS}

I owe my deepest gratitude to my advisor, Dr. Birsen Sirkeci-Mergen, for her sincere support, unlimited encouragement, and meaningful guidance during the whole process from initial research to final thesis reporting. My thesis would not be possible without my advisor. She has always been available and willing to help me. Furthermore, she introduced me to this interesting area of research and helped me perceive happiness here. The thesis inspired me to pursue further research in this field of study. I really appreciate my advisor for all the new pathways of research she has opened.

I am also grateful to my committee members, Dr. Robert H. MorelosZaragoza and Dr. Essam Marouf, for their valuable inputs and for their kindness to accommodate me and make the final defense possible on the day of my choice.

Moreover, I would also like to thank my friends who worked with me and made my research experience very enjoyable. Last but not least, I would like to thank my family for their pure love and understanding. Especially, I would like to thank my husband for all the support he has provided. 


\section{TABLE OF CONTENTS}

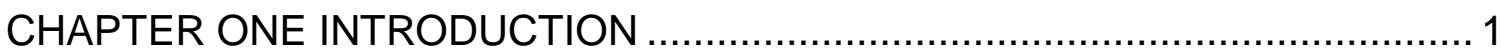

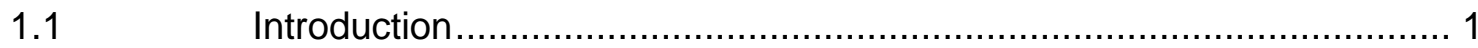

1.1.1 Wireless Channel Impairments ..................................................... 1

1.1.2 Methods to Combat Fading ........................................................... 2

1.1.3 Contributions of This Work.......................................................... 5

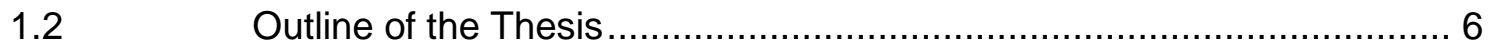

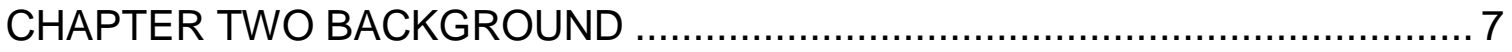

2.1 Overview of Wireless Fading Channels ….................................... 7

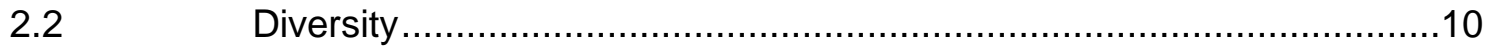

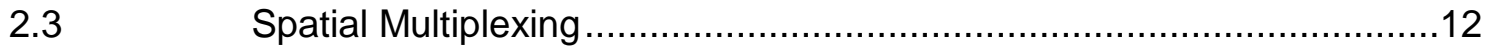

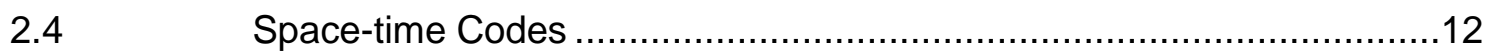

\section{CHAPTER THREE COOPERATIVE SIGNALING FOR SINGLE-ANTENNA}

MULTI-HOP RELAY NETWORKS ........................................................... 14

3.1 System Model of Multi-hop Networks with Single-antenna Relays ........16

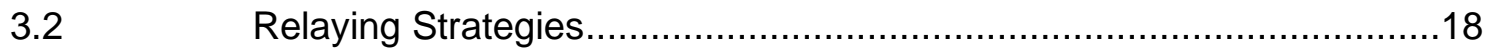

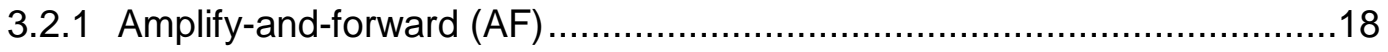

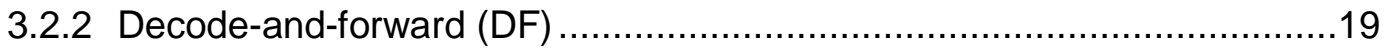

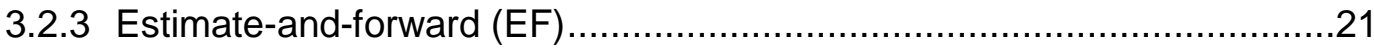

3.2.4 Proposed Relay Strategy: Saturated Amplify-and-forward (SAF) ............23

S.3 Simulations Based on Multi-hop Relay Networks ............................27 
3.3.1 The Effect of Path-loss Exponents on the Performance of Multi-hop Networks

3.3.2 The Effect of the Number of Relays on the Performance of Multi-hop

Networks .29

3.3.3 The Effect of Relay Locations on the Performance of Multi-hop Network 30 CHAPTER FOUR THE EFFECT OF MULTI-ANTENNA ON THE PERFORMANCE OF MULTI-HOP RELAY NETWORKS 31

4.1 System Model of Multi-hop Networks with Multi-antenna Relays ..........31

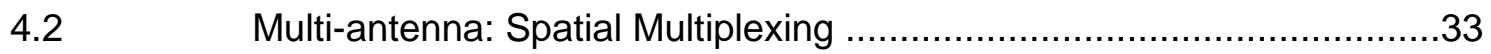

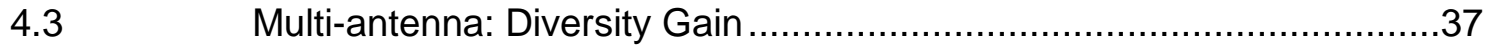

\section{CHAPTER FIVE COOPERATIVE SIGNALING FOR MULTI-STAGE RELAY}

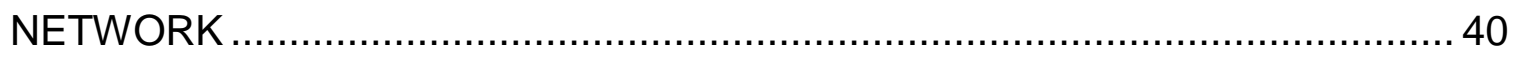

Motivation of Multi-stage Relay Networks ….............................40

System Model of Multi-stage Relay Networks ..................................42

5.3 Relay Processing: Preprocessing, Mapping, and DSTCs...................44

5.3.1 Relay Processing in the First Stage ...............................................44

5.3.2 Preprocessing in Following Stages ............................................48

5.3.3 Optimal Maximum Likelihood Decoding .........................................49

Simulations Based on Multi-stage Relay Networks .........................50

5.4.1 The Effect of Mapping Functions on the Performance of Single Stage Networks 
5.4.2 The Effect of the Stage Locations on the Performance of Multi-stage

Networks

5.4.3 The Effect of the Number of Stages on the Performance of Multi-stage

Networks

CHAPTER SIX CONCLUSIONS AND FUTURE WORK....

6.1

Conclusions

6.2

Future Work.

REFERENCES 


\section{LIST OF FIGURES}

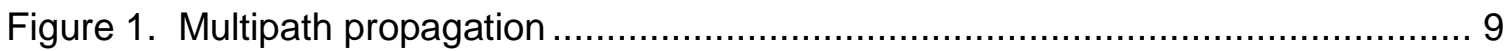

Figure 2. Multi-hop transmission through car to car ....................................................14

Figure 3. A multi-hop single-antenna relay network …….........................................16

Figure 4. Symbol error performance for different modulations with DF scheme ............20

Figure 5. Relay function for AF, DF, MMSE, and SAF .........................................22

Figure 6. Symbol error performance for different relay strategies ...............................24

Figure 7. Optimal symbol error performance for SAF scheme ..................................26

Figure 8. Symbol error performance for different path-loss exponents .........................28

Figure 9. Symbol error performance for different number of relays ............................29

Figure 10. Symbol error performance for different distance allocations........................30

Figure 11. A cooperative relay network with multiple antennae .................................32

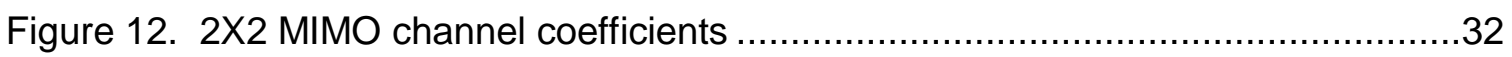

Figure 13. The rule of division and combination signals for $2 \mathrm{X} 2 \mathrm{MIMO} \ldots \ldots \ldots \ldots \ldots \ldots \ldots \ldots \ldots . . .35$

Figure 14. Symbol error performance comparison between MIMO and SISO ...............37

Figure 15. Symbol error performance of MIMO to verify space diversity .......................38

Figure 16. A Cooperative relay network with multiple stages ....................................43

Figure 17. Processing of each relay node in multi-stage relay network.........................45

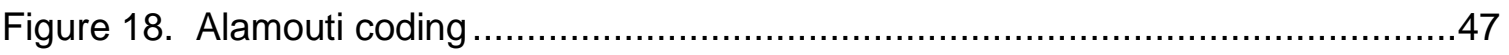

Figure 19. Comparison of mapping function in one stage relay network .......................52

Figure 20. Performance comparison as a function of relay location ..............................53

Figure 21. Comparison of mapping function in a multi-stages relay network................54 


\section{CHAPTER ONE}

\section{INTRODUCTION}

Growing demand for wireless applications has driven significant development of wireless communications. With this development, expectations of reliable wireless applications at high data rate are becoming increasingly exigent. However, wireless channels have several inherent impairments that make achieving high data rate and reliable communications particularly challenging. Therefore, a large effort has been invested to investigate wireless channels and develop novel techniques in order to enhance the wireless communication reliability and to increase the transmission data rate.

\subsection{Introduction}

\subsubsection{Wireless Channel Impairments}

Major wireless channel impairments typically include shadowing, pathloss, and small-scale fading, among which the fading arising from multipath propagation has attracted considerable attention.

Multipath fading results from the rich wireless propagation environment in which transmitted signals may arrive at a destination through different paths and the destination receives multiple copies of the transmitted signals with different phases and magnitudes. If the multiple copies of received signals happen to be in phase, then they can add together constructively to generate a stronger signal 
than the one received from a single path. However, it is equally possible that the different copies of signals are out of phase. Then they may cancel each other producing a weak signal or even no signal. Hence multipath fading yields unpredictable severe time-varying fluctuation in received signals, making reliable communications extremely difficult. We have learned that in additive white Gaussian noise environment, adding 1 or $2 \mathrm{~dB}$ signal-to-noise radio (SNR) can improve bit error rate (BER) from $10^{-2}$ to $10^{-3}$. However, in multipath fading channel it may require up to $10 \mathrm{~dB}$ SNR to achieve the same improvement of BER [1]. Obviously, increasing transmitting power is an effective way to improve the reliability of the system, but not practical in most battery limited wireless applications.

\subsubsection{Methods to Combat Fading}

One of the most powerful techniques to combat the effects of fading is to adopt multiple independent signal paths [2]. By transmitting the same signals over different independent paths, a receiver obtains multiple copies of independently faded replicas of transmitted signals at the destination side. Even though the received signals are faded, they have little chance to be faded at the same time and at the same point since the channels through which they are passing are independent. Then the received signals can be combined in certain ways to generate a stronger resultant signal than the one obtained through a single channel. Accordingly, fading is effectively mitigated. In other words, the reliability can be dramatically improved. 
Another point of view is called spatial multiplexing, which is implemented through sending different independent signals over multiple independent paths. After dividing the source signals into several independent groups and sending each group through each parallel channel, we can easily obtain high data rate transmission.

It is well known that multiple-input multiple-output (MIMO) antennas are one of the most effective methods to achieve both multiplexing gain and diversity gain [3-6]. However, multiple antennae are not affordable for most ad-hoc networks or wireless sensor networks due to limitations in the size, cost, and hardware complexity.

Fortunately, cooperative diversity has been introduced to overcome the limitation in cellular networks [7-10]. Extensive studies have been performed for ad-hoc networks as well [11-15]. In short, cooperative diversity is achieved via distributed multiple single-antenna nodes cooperating with each other to transmit and receive. The multiple single-antenna nodes cooperate with each other to form a virtual multi-antenna system capable of creating multiple independent paths between a transmitter and a receiver as MIMO antennas exhibit.

Another effective way to combat impairments in wireless channels is by the use of relaying techniques that transfer information over multiple relays between source and destination. Relay strategies have been extensively studied [16-18]. Among them decode-and-forward (DF) and amplify-and-forward (AF) schemes are two fundamental strategies for two-hop networks [17]. Recently, 
with the appearance of cooperative diversity, cooperative relaying has attracted considerable attention [13,14]. Meanwhile, distributed space-time coding (DSTC) designed specifically for cooperative relay networks has attracted large attention as well [14]. DSTC can be considered as the extension of space-time coding (STD) to yield additional diversity such that the system performance can be considerably improved.

Currently, the design and analysis of cooperative relay networks using DSTC were usually performed at a high signal-to-noise radio (SNR) regime [1921]. The performance of high SNR outage capacity of cooperative systems, based on repetition or space-time coding, has been studied [13]. The bit error rate behavior of a two-relay system with DSTC in high SNR regime has been analyzed [19]. However, in ad-hoc networks and wireless sensor networks, high SNR is difficult to achieve for relay nodes due to battery limitations. Moreover, most of the previously proposed relaying schemes permit only the relays that decode source signals correctly to retransmit, which can be applied to high SNR regime properly. But for a low-to-moderate SNR regime, the number of relays that can decode signals successfully may be very small, even absent. As a result, there will be a few or no nodes that can perform retransmission. This performance demonstrates that the strategy does not work for a low-to-moderate SNR regime. There have been several new schemes proposed to overcome the drawbacks [22,23]. A linear dispersion code is utilized plus a linear scaling of the received signals instead of decoding at the relays all together [22]. Another 
method introduced is to combine the influence of decoding error into the analysis of DSTCs [23].

\subsubsection{Contributions of This Work}

A novel scheme, called the saturated amplify-and-forward (SAF) scheme, which is a generalization of well known decode-and-forward (DF) scheme and amplify-and-forward (AF) scheme, has been proposed by Birsen Sirkeci-Mergen in 2008 [24]. Simulation results have shown that the SAF scheme followed by distributed space-time coding can outperform the current AF and DF strategies, especially in a low-to-moderate SNR regime. However, the work and the analysis are merely performed based on a single stage relaying network.

In this work, the primary contribution is the development of the new relaying scheme, SAF scheme, for multi-stage networks using symbol-level modulations. More specifically, we extend single stage relaying networks [24] to multi-stage relaying networks, based on which the novel scheme, SAF scheme, followed by space-time coding is analyzed and explored. In addition, the optimal stage allocation and the optimal number of relay stages are simulated and discussed. Furthermore, the channel between source and relay is upgraded to a flat fading Rayleigh channel instead of pervious AWGN channel and a path-loss propagation model is added to the multi-stage network model to make the simulations and analyses more practical. 


\subsection{Outline of the Thesis}

The thesis is organized as follows. Chapter 2 provides background and theoretical analysis of wireless channel, spatial diversity, and spatial multiplexing. Chapter 3 describes a fairly fundamental model of multi-hop relay network comprised of multiple serially organized single-antenna relays to analyze cooperative signaling. In addition, the saturated amplify-and-forward (SAF) strategy, a new relay strategy is developed based on the current two famous relay strategies, AF and DF. Chapter 4 extends the multi-hop network model from single-antenna relays to multi-antenna relays. By comparing the system performance of a multi-antenna relays network with the performance of a singleantenna relay network, we discuss the tradeoff between spatial diversity and spatial multiplexing. Chapter 5 is the main novel part of this study. It extends the basic single-antenna multi-hop network to a multi-stage relay network with each stage containing several single-antenna nodes, in which the newly proposed strategy, SAF strategy, used with space-time coding is simulated and analyzed. In addition, the optimal stage allocation and the optimal number of relay stages are demonstrated. Ultimately, Chapter 6 draws overall conclusions and points out directions of future research. 


\section{CHAPTER TWO}

\section{BACKGROUND}

This chapter provides a detailed explanation of wireless propagation environment, which is of great significance in studying wireless relaying networks. In particular, the chapter provides a brief overview of wireless channel, space diversity, spatial multiplexing, and space-time coding. Understanding wireless channels is of vital importance to analyze and solve the problems in wireless channels. Space diversity can effectively combat multipath fading of wireless channels so that reliability of wireless communications can be improved, but it cannot increase transmission data rates. Spatial multiplexing is a great method to increase transmission data rate, however, it cannot improve reliability. Space-time coding presents a method to obtain two objectives, improving reliability and increasing data rates. These topics are fundamentals of this study described in the following chapters.

\subsection{Overview of Wireless Fading Channels}

It has been well established that wireless channels suffer from many impediments, leading to unpredictable and time-varying variations in the received signals. These distortions generally can be divided into two categories, large- 
scale propagation effects and small-scale propagation effects, based on the distance over which variations occur.

Basically, large-scale propagation effects refer to variations that occur over large distances. The variations are usually due to path-loss and shadowing [25]. Path-loss, as the name implies, is the attenuation of transmission power due to distance travelled by the signal. Shadowing is the attenuation of transmission power due to absorption, reflection, scattering, and diffraction of obstacles between a transmitter and a receiver. In our study, to simplify the large-scale propagation model without loss of generality, we adopt a simplified path-loss model, in which transmission power decays with distance proportional as $d^{\alpha}$. The parameter $\alpha$ is the path-loss exponent that is usually between 2 and 4. When $\alpha=2$, the model stands for the free space path-loss model in which no obstruction is located between a transmitter and a receiver. If obstacles are involved, path-loss attenuates more rapidly with distance.

Small-scale propagation effects refer to variations that occur over short distances. These variations are usually due to the constructive and destructive additions of multipath fading signals [25]. It is usually used to describe rapid variations of the amplitudes, phases, or delays of received signals over short period of time or distance. There are several significant factors that influence the small-scale propagation, such as multipath fading, Doppler frequency shift, and bandwidth of transmitted signals. Among them, multipath fading is one of the main topics in this study so it will be discussed in more detail. Multipath fading 
occurs in most wireless channels where multipath propagation exists, when any element moves, or when surrounding environment changes. Multipath propagation describes a common phenomenon in wireless channels. In this case, transmitted signals reach the destination through not only the light of sight (LOS) path but also any other possible paths due to reflection, scattering, or diffractions of obstructions, as shown in Figure 1.

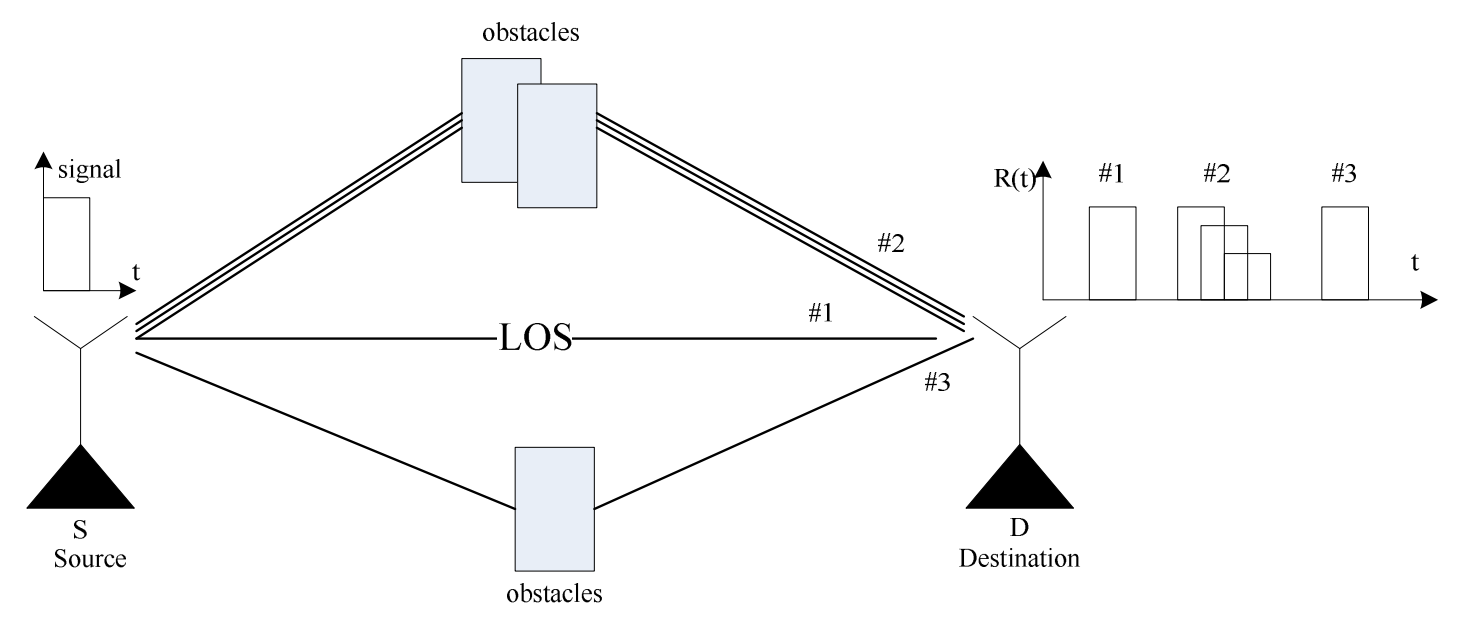

Figure 1. Multipath propagation

Therefore, the destination receives multiple faded replicas of transmitted signals that carry different magnitudes, different phases, and different time delays. These differences are difficult to be measured or predicted since they change with time and environment. Those unpredictable factors may lead to the overall received signal completely faded away at some points in time. They also may cause the power of the received signal increase considerably at other points 
in time. Obviously, the unpredictable and time-varying fluctuations make wireless channel extremely unreliable.

Theoretically, multipath fading is usually described in statistical ways since the geometry and dielectric properties of wireless propagation environment are unknown, especially when the number of multipath components goes to large [25]. In order to exploit the channel model we use in our study, an important channel parameter, coherence bandwidth, is introduced. Coherence bandwidth is used is to measure the range of frequencies over which the channel passes all spectral components with approximately same gain and linear phase. If the coherence bandwidth is larger than the symbol bandwidth, the fading channel can be treated as flat fading. In this study, we adopt a Rayleigh flat fading channel model. We also assume that channel coefficients corresponding to different paths are independent and identically distributed.

\subsection{Diversity}

Multipath fading makes reliable communication in wireless networks hardly achievable. One effective way to solve this problem is to employ diversity. Diversity can be considered as a scheme that uses multiple independent paths to transfer the same signals. The idea is that through a single path received signals may suffer deeply fading easily, but it is rarely possible that received signals through other independent paths are deeply faded as well. Hence, combination 
of these independently faded received signals can mitigate multipath fading effectively.

In wireless channels, there are three commonly used diversity types: time diversity, frequency diversity, and space diversity. The general idea behind time diversity and frequency diversity is to transfer the same signals at different time slots or different carrier frequencies. However, they both have drawbacks. Time diversity consumes extra transmission time and frequency diversity needs extra bandwidth. Fortunately, space diversity can eliminate these shortcomings. For space diversity, implementing multiple antennae is desirable since multiple antennae can generate space diversity naturally. When multiple antennas are placed at the transmitter side, transmit diversity is achieved. When multiple antennae are placed at the receiver side, receive diversity is achieved. Apparently, multi-input multi-output (MIMO) antennas system covers them both. Assuming a MIMO system contains $n$ antennas at the transmitter side and $m$ antennas at the receiver side. Each pair of transmitter antenna and receiver antenna builds one channel. Accordingly, in the system, there will be $m \times n$ different independent channels between the transmitter and the receiver. If we transmit the same signals through $n$ transmitter antennas using repetition scheme, then the transmitted signals will pass through the $m \times n$ independent channels, arriving at the receiver. The receiver then obtains $m \times n$ copies of signals, which are certainly more reliable than one copy of signals received from merely one channel. The improvement of reliability is directly proportional to the 
diversity gain. The maximum diversity gain for the MIMO system with $m$ transmit and $n$ receive antennas is $m \times n$ [27].

\subsection{Spatial Multiplexing}

Spatial multiplexing plays the same important role as space diversity does in wireless channels. The main advantage of using spatial multiplexing is to increase transmission data rate. Multiple antennae are still essential because of the degree of freedom they provide. Contrary to space diversity, spatial multiplexing transmits different signals through each of the multiple transmitter antennas. The motivation of the approach is that the channel matrix of multiple antennae system can be reconstructed to parallel spatial channels [27]. Therefore, different signals are transmitted through parallel channels and hence the data rate can be increased tremendously. Transmission data rate increases in direct proportion to the number of degree of freedom. In a MIMO system with $m$ transmit and $n$ receive antennas, there are $\min \{n, m\}$ degrees of freedom [27].

\subsection{Space-time Codes}

In Section 2.2, it has been exploited that transmitting the same signals through multiple antennae could achieve maximum space diversity gain $n \times m$. However, the transmission data rate in that case is equal to $1 / n \mathrm{symbol} / \mathrm{s} / \mathrm{Hz}$. In Section 2.3, the data rate can achieve $\min (n, m)$ symbol/s/Hz by using multiplexing. However, the diversity gain is only 1 , which is the same as the gain 
of a single-antenna system. Fortunately, space-time coding offers the opportunity to achieve both diversity and high data rate. Generally, the idea behind space-time coding is not only transmitting several symbols at the same time but also obtaining diversity. There have been intensive studies making contributions to this area. Among them, Alamouti space-time coding invented by Alamouti in 1998 is a simple and powerful space-time coding [1].

Alamouti space-time coding offers a method to achieve space diversity by two antennas. Instead of transmitting symbols one by one, it groups the intended transmitting symbols into groups of two. During the first time slot, two symbols in one group, $s_{1}$ and $s_{2}$ are transmitted through the first and the second antenna respectively. At the second time slot, $-s_{2}^{*}$ and $s_{1}^{*}$ are transmitted through the first and the second antenna respectively. Here $*$ is the conjugate operator. Therefore, two symbols are sent out during two time slots, which generates maximum data rate of $1 \mathrm{symbol} / \mathrm{s} / \mathrm{Hz}$ that doubles the rate of repetition scheme in Section 2.2. Nevertheless, the diversity gain is 2 , which is the full diversity of $2 \times 1 \mathrm{MIMO}$ system. Thus, the advantages of Alamouti space-time coding are obvious. 


\section{CHAPTER THREE}

\section{COOPERATIVE SIGNALING FOR SINGLE-ANTENNA MULTI-HOP RELAY NETWORKS}

This chapter focuses on cooperative signaling strategies in multi-hop networks formed by a single source-destination pair and multiple single-antenna relays. Transmissions between the source and the destination are assumed to be in a multi-hop fashion. As the name implies, multi-hop networks adopt two or more wireless hops to transmit signals from a source to a destination. One transmission between a transmitter and a receiver is called one hop. As shown in Figure 2, a vehicular network is presented as an example.

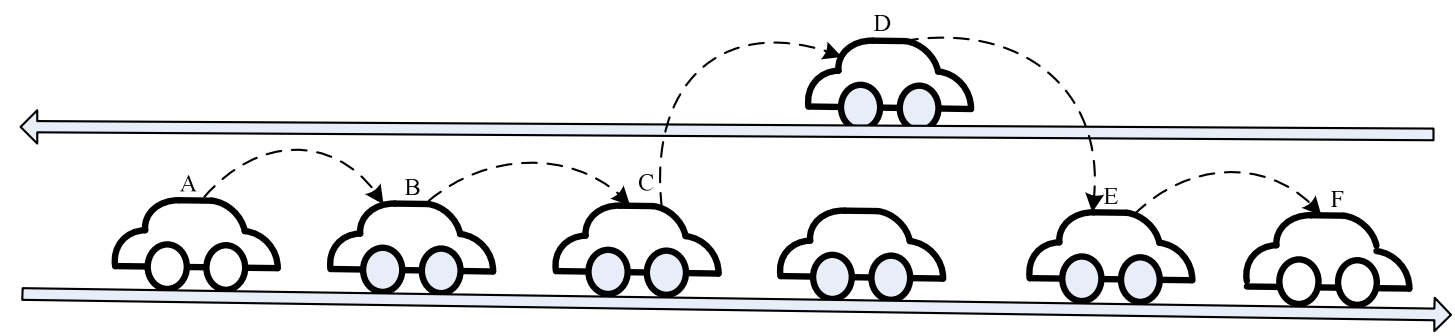

Figure 2. Multi-hop transmission through car to car

Figure 2 presents a vivid example of transmission processes in a multihop network. Assume car $A$ holds the source signals and $\operatorname{car} F$ is the destination. At first, car $A$ transmits signals to car $B$. Then car $B$ retransmits the signals to car $C$. Following this procedure, the signals eventually arrive at car $F$ after multi-hop. 
In this chapter not only will the performances of amplify-and-forward (AF) and decode-and-forward (DF) be analyzed based on the multi-hop network, but also a novel relay strategy will be proposed. It is worth noting that the relaying we focus on is based on symbol-by-symbol relaying. It has been well established that amplify-and-forward (AF) and decode-and-forward (DF) are the two most popular cooperative relaying schemes in single relay systems [28]. As the name suggests, AF scheme permits the relay node to amplify the received signals to a certain power level and retransmit them. DF scheme allows the relay node to decode the received signals, re-encode them, and then retransmit them. However, both schemes have their own weak points. For AF scheme, the noise is amplified while the signals are being amplified. For DF scheme, the network performance depends seriously on the worst link performance. Therefore, in order to overcome the drawbacks, we study a new scheme called saturated amplify-and-forward (SAF) [24] in a multi-hop setup, and the simulation results show that the proposed scheme outperforms both AF and DF.

In the following, Section 3.1 describes the basic system model of a multihop network. Section 3.2 analyzes several well known relaying strategies, such as $\mathrm{AF}, \mathrm{DF}$, and estimate-and-forward (EF). In addition, the novel scheme SAF is developed. During the discussion of DF scheme, three symbol-level modulation methods, BPSK, QPSK, and DBPSK, are analyzed and compared. At the end, Section 3.3 presents several simulations based on this system model to verify the advantage of the newly proposed scheme in multi-hop networks. 
3.1 System Model of Multi-hop Networks with Single-antenna Relays

In this section, the system model is highlighted. We consider a cooperative multi-relays network formed by a single source $S$, a single destination $D$ and a group of $M$ relays $\left\{R_{1} \cdots R_{i} \cdots R_{M}\right\}$ which are serially arranged. All the nodes carry only one antenna, as shown in Figure 3. We also consider that the time is slotted. In each slot, source or only one of the relays is active, as shown in Figure 3 as well. Accordingly, the communication process can be simplified as, at any time slot, only one node transmitting and only the subsequent node receiving.

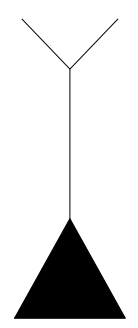

$\mathrm{S}$

Source

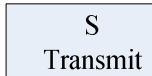

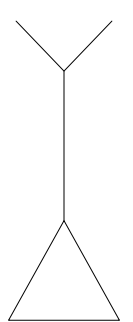

$\mathrm{R}_{\mathrm{l}}$

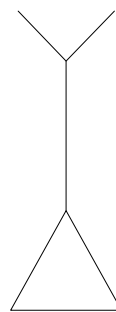

$\mathrm{R}_{2}$

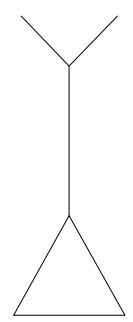

$\mathrm{R}_{\mathrm{M}}$

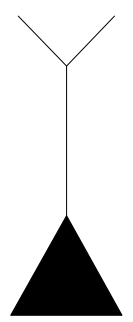

D

Destination

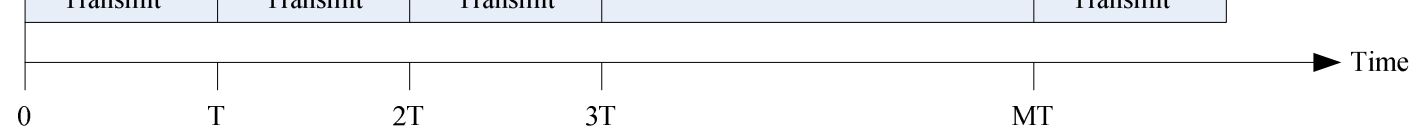

Figure 3. A multi-hop single-antenna relay network

It is also assumed that a simplified path-loss model is adopted for all propagations between the source and relay, between relay and relay, and between relay and the destination. 
The simplified path-loss model can be written as

$$
\sigma_{i}^{2} \propto \frac{1}{d^{\alpha}}
$$

Equation 1

where $\sigma_{i}^{2}$ is the variance of the path-loss power in the $i$ 'th link between $R_{i-1}$ and $R_{i}, d$ is the distance of the $i$ 'th link, and $\alpha$ is the path-loss exponent which is usually taken from 2 to 4 , depending on the propagation environments.

Let $\bar{s}=\left[s_{1} \cdots s_{N}\right]$ stand for the transmitted symbols by the source $S$ with average power $P_{S}=E\left\{|\bar{s}|^{\wedge} 2\right\} . E\{\cdot\}$ means the expectation operator and $N$ is the total number of symbols transmitted from the source. Let $\bar{r}^{[i]}=\left[r_{1}{ }^{[i]} \cdots r_{N}{ }^{[i]}\right]$ denote the received signals at the $i$ 'th relay and $\bar{h}^{[i]}=\left[h_{1}{ }^{[i]} \cdots h_{N}{ }^{[i]}\right]$ denote the Rayleigh channel coefficients during the $i^{\prime}$ th link. At the first relay, the received signals can be written as

$$
\bar{r}^{[1]}=\bar{s} \cdot * \bar{h}^{[1]}+\bar{n}^{[1]}
$$

Equation 2

where $\bar{n}^{[1]} \in \bar{n}^{[i]}=\left[n_{1}{ }^{[i]} \cdots n_{N}{ }^{[i]}\right]$ are zero-mean complex additive white Gaussian noise (AWGN) with variance $\sigma_{n}^{2}=N_{0} / 2$ in the first link. The channels are assumed to be Rayleigh flat fading, so $\bar{h}^{[1]} \in \bar{h}^{[i]}=\left[h_{1}{ }^{[i]} \cdots h_{N}{ }^{[i]}\right]$ are complex Gaussian random variables with zero mean and variance $1 / 2$. Channel state information (CSI) is available at the receiver in general. 


\subsection{Relaying Strategies}

\subsubsection{Amplify-and-forward (AF)}

Amplify-and-forward strategy is explained in this subsection [28]. In the first time slot, the source $S$ broadcasts signals and the first relay $R_{1}$ receives the signals $\overline{y_{R_{1}}}=\bar{r}^{[1]}$ where $\bar{r}^{[1]}$ can be obtained from Equation 2 .

At the second time slot, the relay $R_{1}$ amplifies the received signals $\overline{y_{R_{1}}}$ to its power level and retransmits.

The retransmitted signals $\overline{t_{R_{1}}}$ are

$$
\overline{t_{R_{1}}}=\sqrt{\frac{P_{1}}{P_{R_{1}}}} \overline{y_{R_{1}}}
$$

where $\sqrt{P_{1} / P_{R_{1}}}$ is used to amplify the power of received signals to the given power level $P_{1}$. $P_{R_{1}}$ can be written as $P_{R_{1}}=E\left\{\left|\overline{y_{R_{1}}}\right|^{\wedge}\right\}$.

Consequentially, it is reasonable to generalize the relay rule to

$$
\overline{t_{R l}}=\sqrt{\frac{P_{i}}{E\left\{\left.\overline{\bar{y}_{R_{l}}}\right|^{\wedge}\right\}}} \overline{y_{R l}}
$$

in which $\overline{y_{R_{l}}}$ and $\overline{t_{R_{1}}}$ represent the received signals and retransmitted signals at the $i$ 'th relay respectively. $\sqrt{P_{i} / E\left\{\left|\overline{y_{R_{l}}}\right|^{\wedge}\right\}}$ is used to amplify the power to the given power limit $P_{i}$ at $i$ 'th relay. 


\subsubsection{Decode-and-forward (DF)}

As for decode-and-forward scheme, it goes through a bit more complicated process than AF since it involves two processes, decode and reencode. In this situation, the received signals at the $i$ 'th relay $\overline{y_{R_{l}}}$ and the retransmitted signal $\overline{t_{R_{l}}}$ depends on the modulation type. Below we will discuss three different modulation schemes: BPSK, QPSK, and DBPSK.

BPSK, called binary phase shift keying, is the simplest modulation in phase shift keying. It adopts two phases and one phase stands for "1" and another phase stands for " 0 ", and the two phases separate from one another by $\pi$. Quadrature phase shift keying (QPSK) has four phases equally distributed in coordinate plane with each phase mapping to two transmitting bits. Differentially binary phase shift keying (DBPSK) is using phase differences to encode the transmitted signals, rather than the phase itself to encode the transmitted signals. So the receiver can demodulate received signals successfully even though the receiver does not estimate the carrier phase exactly [29].

The performance of these schemes for a point-to-point communication is well known. It has been proven that the BPSK performance on the AWGN channel with zero mean and variance $N_{0} / 2$ can be written as $P_{S}=Q\left(\sqrt{2 \mathrm{E}_{S} / N_{0}}\right)$ in which $P_{S}$ is the probability of symbol error and $E_{S} / N_{0}$ is the received signal-tonoise ratio (SNR) per symbol. $Q(x)$ is the function to calculate the probability of a standard normal random variable obtaining a value larger than $x$. It can be 
demonstrated as $Q(x)=\frac{1}{2 \pi} \int_{x}^{\infty} e^{-u^{2} / 2} d u$. For QPSK, the probability of bit error is identical to the one of BPSK because QPSK can be treated as two quadrature BPSKs. However, QPSK needs to spend twice the power of BPSK to achieve the same bit error rate. For DBPSK, the noise variance is two times as large as the one of BPSK, so its performance can be considered as $3 \mathrm{~dB}$ poorer than that of BPSK [29].

The same behavior is valid for a single relay network as shown in Figure 4. We place the source and destination $10 \mathrm{~m}$ apart and the relay at the middle. The signal attenuates based on the free path-loss model with path-loss exponent $\alpha=2$. Each node transmits with same unit power. A flat Rayleigh fading channel is considered.

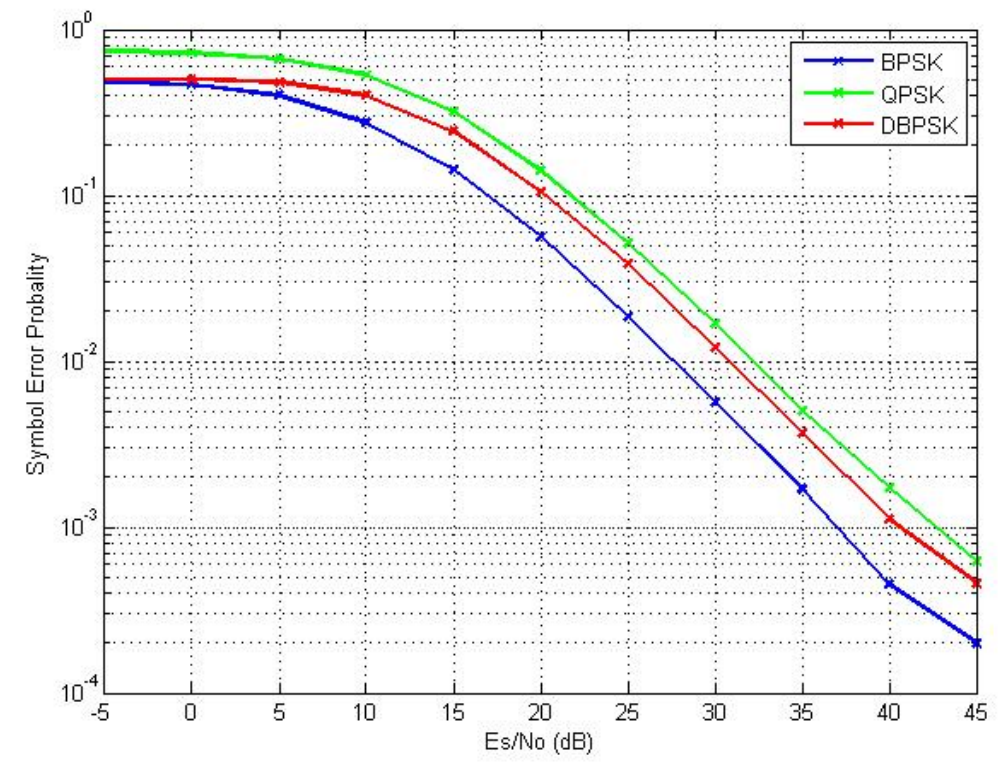

Figure 4. Symbol error performance for different modulations with DF scheme 
As shown is Figure 4, BPSK has the lowest symbol error rate compared with QPSK and DBPSK. As expected, the same behavior is valid for the single relay multi-hop network.

For simplicity, we will adopt BPSK in the following as decode-and-forward scheme. In this situation, the received signals at $i$ 'th relay is $\overline{{y_{l}}_{l}}$ and the retransmitted signals $\overline{t_{R_{l}}}$ can be written as

$$
\overline{t_{R l}}=\sqrt{P_{i}} \operatorname{sign}\left(\operatorname{Re}\left(\overline{y_{R l}}\right)\right) \quad \text { Equation } 5
$$

The Equation 5 presents the relationship between relay input and relay output for decode-and-forward scheme.

\subsubsection{Estimate-and-forward (EF)}

A minimum mean square error (MMSE) based estimate and forward (EF) scheme has been used in relay networks [30]. It concluded that in memoryless relay network, the EF scheme can satisfy the characterization of the optimal relay function in the single relay system. It also concluded that in multi-relay systems, both serial and parallel case, the new scheme outperforms AF and DF but with a small gain.

The motivation behind the new scheme is that $\mathrm{AF}$ and $\mathrm{DF}$ both have their own weak points as discussed in Section 2.3.2 and Section 2.3.3. The DF relay scheme requires decoding and then re-encoding. In the process, all the inputs are decoded symbol by symbol with hard decision, which generates errors easily. And if any of the relays in a multi-hop setup makes some wrong decision, the errors will be passed to the following nodes until the destination. The EF method 
can add certain soft information when it makes decoding decisions [30]. For AF scheme, Equation 4 shows that it demands considerable energy to scale, especially when $\left|\overline{{y_{l}}_{l}}\right|$ is high. The EF can properly limit the energy consumed. It can be easily shown that the relay function of EF scheme with BPSK modulation simplified as

$$
\overline{t_{R_{l}}}=\sqrt{\frac{P_{i}}{E\left(\mid \tanh \left(\left.\sqrt{\left.\overline{{\overline{R_{-}}}_{2}}\right)}\right|^{\wedge}\right)\right.}} \tanh \left(\overline{y_{R_{l}}}\right)
$$

Equation 6

Details of this derivation are shown in [30].

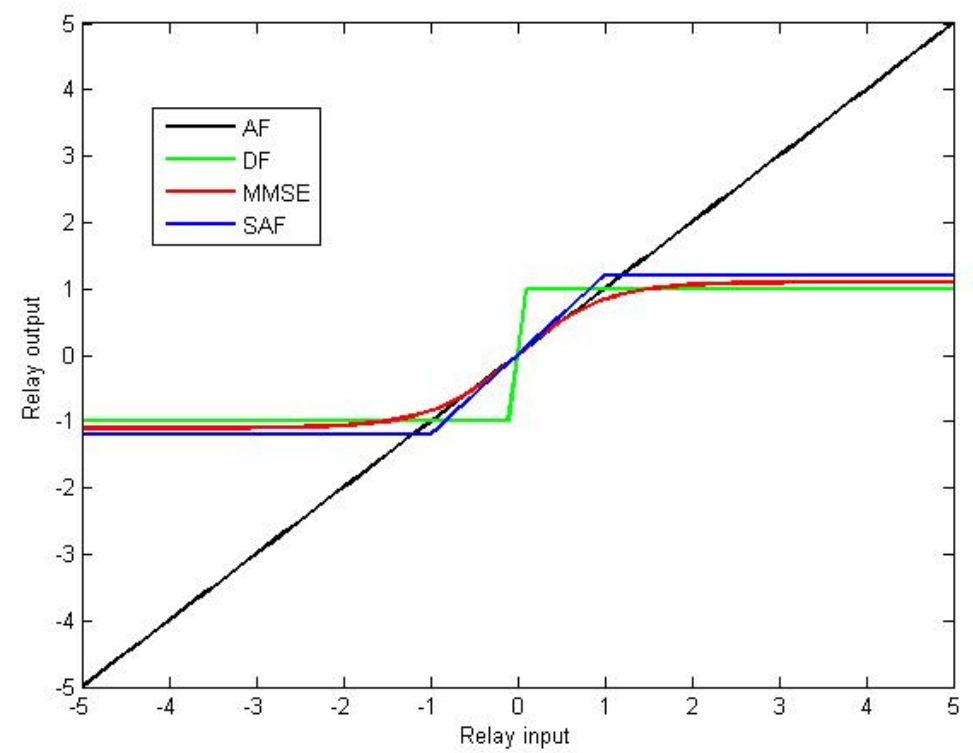

Figure 5. Relay function for AF, DF, MMSE, and SAF

In order to demonstrate the differences among these schemes, Figure 5 is created. Figure 5 presents the relationship between relay input and output for all three schemes discussed (AF,DF, MMSE EF) in addition to the newly proposed scheme saturate AF for BPSK modulated symbols (see Section 3.2.4). 
3.2.4 Proposed Relay Strategy: Saturated Amplify-and-forward (SAF)

We propose a new parameterized relay function so that we can search for the optimal parameter to obtain the premium system performance. The scheme is called saturated amplify-and-forward that is first introduced in [24].

The relay function at $i$ 'th relay is given as

$$
\overline{Z_{R_{l}}}=\left\{\begin{array}{cc}
1 & \operatorname{Re}\left(\overline{y_{R_{l}}}\right)>\tau \\
-1 & \operatorname{Re}\left(\overline{y_{R_{l}}}\right)<-\tau \\
\frac{\operatorname{Re}\left(\overline{y_{R_{l}}}\right)}{\tau} & \text { o.w. }
\end{array}\right.
$$

in which $\tau$ is the controllable parameter and $\operatorname{Re}(\cdot)$ means the real part operator.

As a result the transmitted signals are

$$
\overline{t_{R_{l}}}=\sqrt{\frac{P_{i}}{E\left\{\left.\overline{Z_{R_{l}}}\right|^{\wedge}\right\}}} \overline{Z_{R_{l}}}
$$

in which $\sqrt{P_{i} / E\left\{\left|\overline{Z_{R_{l}}}\right|^{\wedge}\right\}}$ is to normalize the power to the given power limit $P_{i}$.

Obviously, when $\tau$ equals to 0 , the relay function is equivalent to

$$
\overline{Z_{R_{-l}}}=\left\{\begin{array}{cc}
1 & \operatorname{Re}\left(\overline{y_{R_{i}}}\right)>0 \\
-1 & \operatorname{Re}\left(\overline{y_{R_{i}}}\right)<0
\end{array}\right.
$$

which is totally the same as $\operatorname{sign}(\cdot)$ function that is the relay function of DF scheme for BPSK modulation. When $\tau$ goes towards infinite, the relay function can be written as

$$
\overline{Z_{R_{l}}}=\frac{\operatorname{Re}\left(\overline{y_{R_{l}}}\right)}{\tau}
$$


Let $\sqrt{P_{i} / E\left\{\left|\overline{y_{R_{l}}}\right|^{\wedge}\right\}}=1 / \tau$, the relay function is exactly the same as the one of AF (Refer to Figure 5).

In order to confirm the above conclusion, we consider a two-hop network based on the model in Section 3.1. BPSK modulation is adopted and nodes transmit with equal power. The distance between source and destination is 10 meters. One relay moving from source to destination with step of 2 meters and $D_{1}$ is the distance between source and the relay. Path-loss exponent $\alpha$ is equal to 2.5. The simulation is conducted at a fixed symbol noise radio $E_{S} / N_{0}=30 \mathrm{~dB}$. The simulation is done through MATLAB.

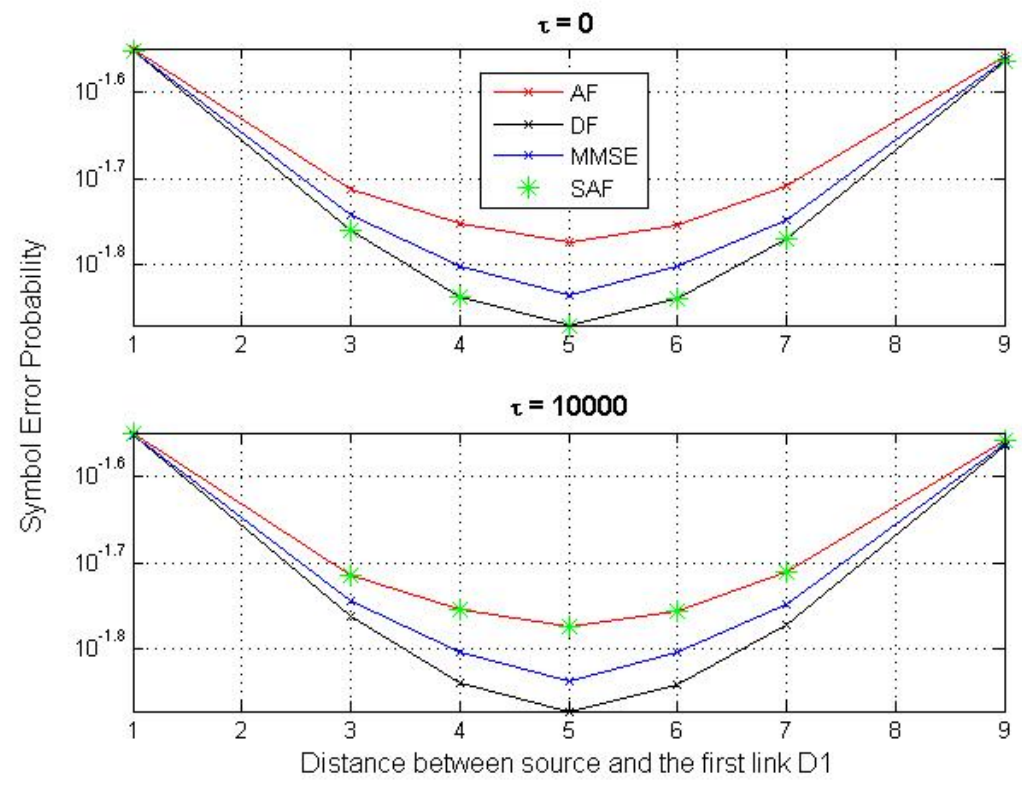

Figure 6. Symbol error performance for different relay strategies

Figure 6 confirms that when $\tau=0$ the saturated amplify-and-forward (SAF) generates the identical effect as DF does on the performance. When 
$\tau=10000$ the SAF generates the same effect as AF does on the performance. Furthermore, placing the relay in the center of source and destination is optimal for all schemes based on the above model.

According to the above analysis, when the flexible parameter in the saturated AF scheme is selected optimally, the scheme can outperform AF, DF, and estimate and forward in especially general network architecture, not necessarily multi-hop.

In order to obtain the optimal parameter, exhaustive search method is employed. We let $\tau$ increase from 0 to 20000 with different steps. In the small value range from 0 to 2 , a small increase step 0.05 is chosen, while in the large value range from 2 to 20000 , a large increase step 500 is chosen. For each $\tau$, a result of symbol error probability is obtained. After 80 times of tests, we will collect 80 probabilities of symbol error. Among them, we choose the lowest value as the best performance. The corresponding $\tau$ is the optimal $\tau$. Based on the same model and the same specifications by which Figure 6 is obtained, the optimal performance is displayed in Figure 7. 


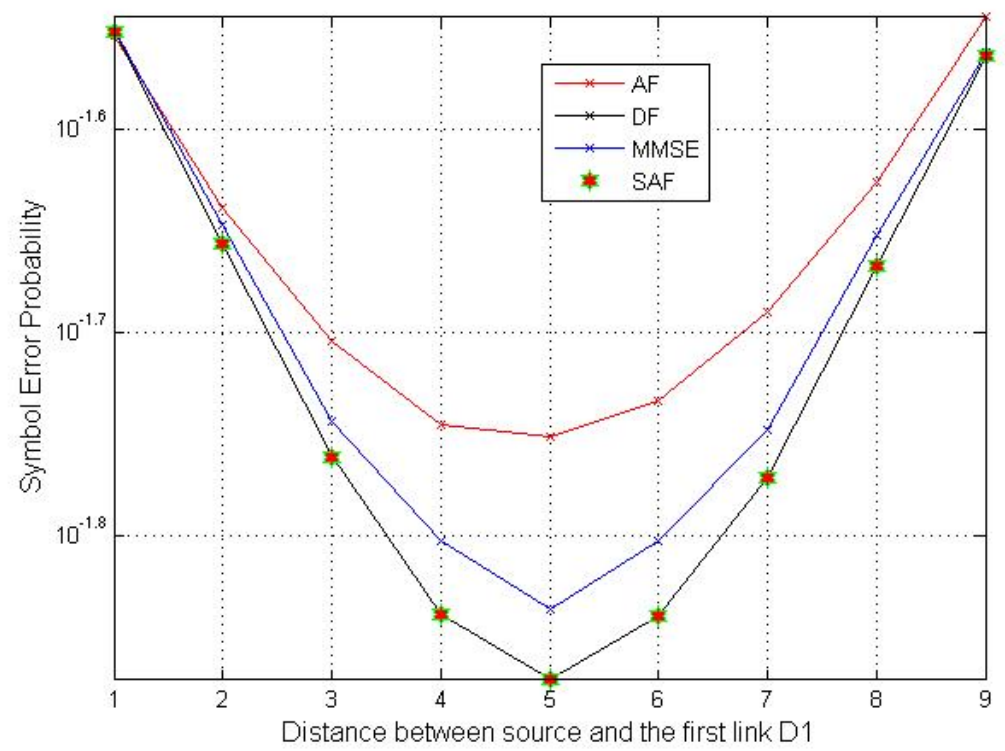

Figure 7. Optimal symbol error performance for SAF scheme

Figure 7 presents that the optimal performance of SAF, which is the same as the performance of decode-and-forward (DF). It means that in a multi-hop network, the performance obtained by DF is the optimal. The results are actually well known in the case when relays are allowed to use complicated channel codes [31].

Basically the performance gain of SAF comes with increased complexity. In addition, it is important to note that the parameter $\tau$ depends on channel gains of all links, which means global channel state information (CSI) is needed when SAF is adopted. 


\subsection{Simulations Based on Multi-hop Relay Networks}

In this section, system performances are evaluated under different situations through MATLAB. The system model in Section 3.1 is adopted. The distance between source and destination is set to $10 \mathrm{~m}$, and the source and relays use BPSK modulation. All nodes transmit with equal unit power. Flat Rayleigh fading is considered, and channel coefficients $\bar{h}^{i}$ in every link are independent and identically distributed. The variance of the AWGN noise at the relays and destination is denoted by $N_{0}$. The performance is demonstrated by the curve of the symbol error probability as a function of symbol noise radio $E_{s} / N_{0} . E_{S}$ is the average power of transmitted signals in the source. All three schemes, AF, DF, and SAF, are implemented individually based on the specification.

3.3.1 The Effect of Path-loss Exponents on the Performance of Multi-hop Networks In order to observe how the path-loss exponent affects the system performance, we assume an array of different path-loss exponents from 2 to 4 . The number of relays $M$ is equal to 4 . Three schemes, DF, AF, and SAF, are respectively implemented and the results are plotted in the same figure. 


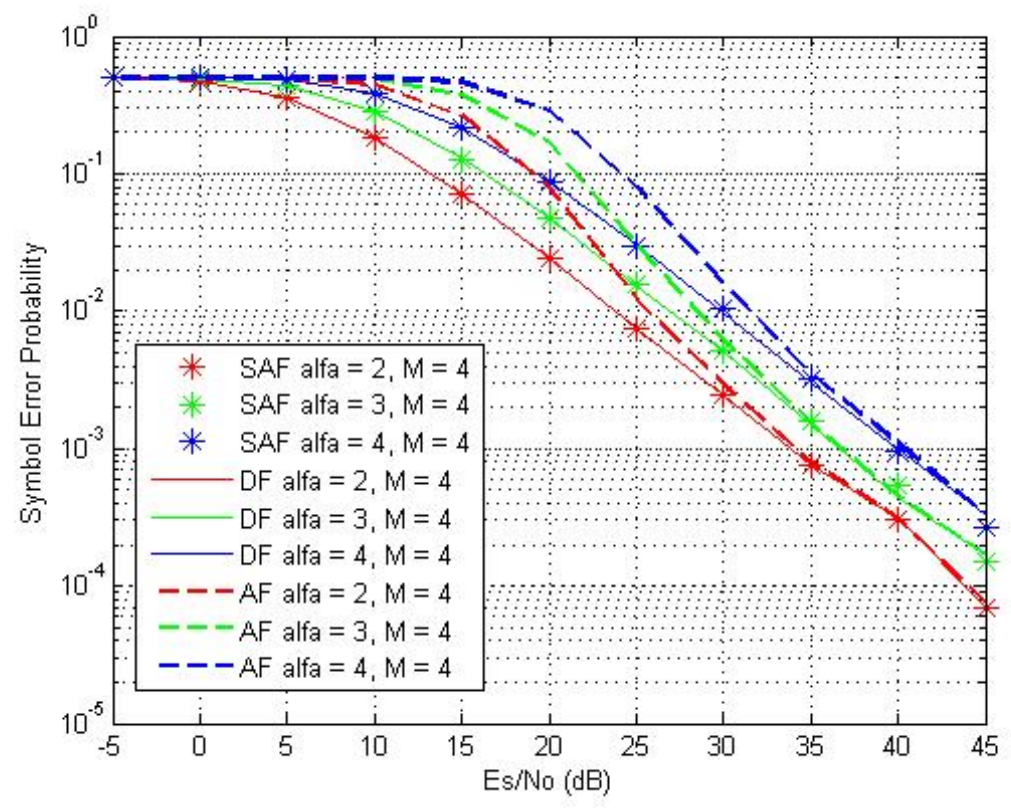

Figure 8. Symbol error performance for different path-loss exponents

In Figure 8, path-loss exponent $\alpha$ is represented by alfa. It is worth noting that the symbol error achieves lowest rate when $\alpha=2$ for all three schemes. As $\alpha$ increases, the symbol error performance becomes worse. As we know, $\alpha=2$ stand for the free space path-loss model. Therefore, we can confirm that free space path-loss is capable of obtaining the best system performance in the exponent range we assigned. Another significant point is that the three schemes perform identically in $E_{s} / N_{0}$ regime larger than $35 \mathrm{~dB}$. However, in the lower $E_{S} / N_{0}$ range, SAF performs the same as DF but better than $\mathrm{AF}$. 
3.3.2 The Effect of the Number of Relays on the Performance of Multi-hop Networks

The objective is to evaluate how the number of relays between source and destination affects the system performance. We assume an array of different number of relay from 2 to 6 and path-loss exponent $\alpha=2.5$.

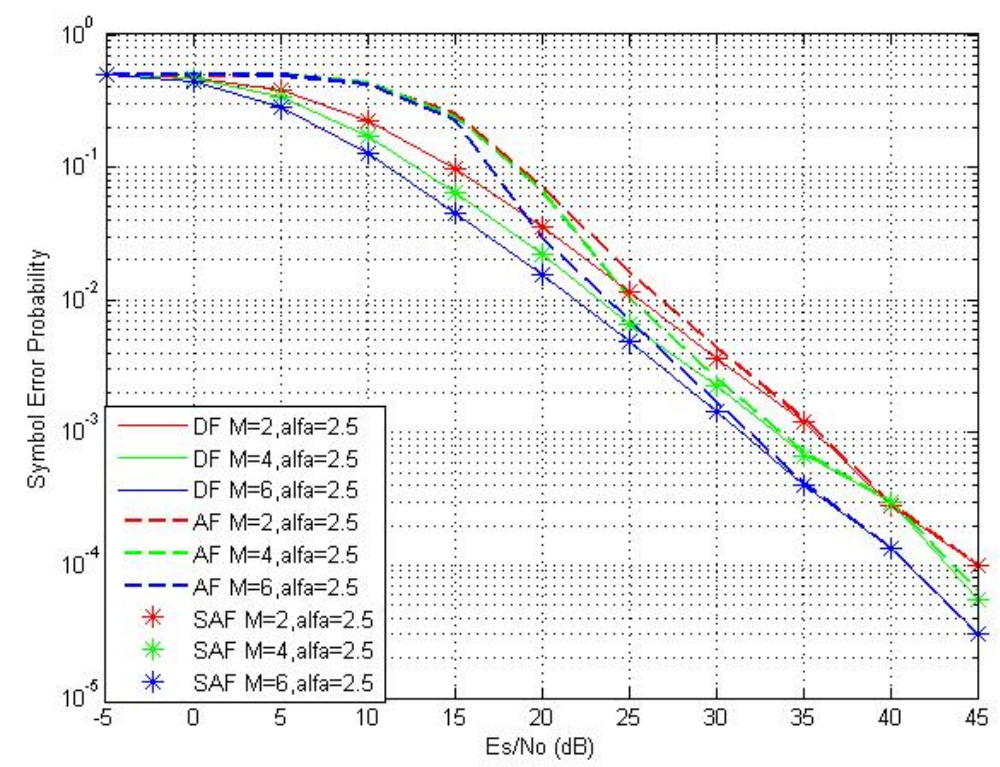

Figure 9. Symbol error performance for different number of relays

In Figure 9, it is obvious to see that as the number of relays increases, the performance becomes better for SAF and DF schemes, which is true for AF in a higher $E_{s} / N_{0}$ range from $15 \mathrm{~dB}$ to $45 \mathrm{~dB}$. It also shows that the three schemes perform identically in $E_{S} / N_{0}$ regime larger than $35 \mathrm{~dB}$. However, in the lower $E_{S} / N_{0}$ range, SAF performs the same as DF but better than AF. 
3.3.3 The Effect of Relay Locations on the Performance of Multi-hop Networks The simulation is to testify how the relay location affects the performance. Assume a two-hop wireless network with a relay located at $D 1$ meters away from the source. Set $D 1=[2,5,8]$ respectively and plot a group of symbol error probabilities based on different distance of the first link. The path-loss exponent is 2 .

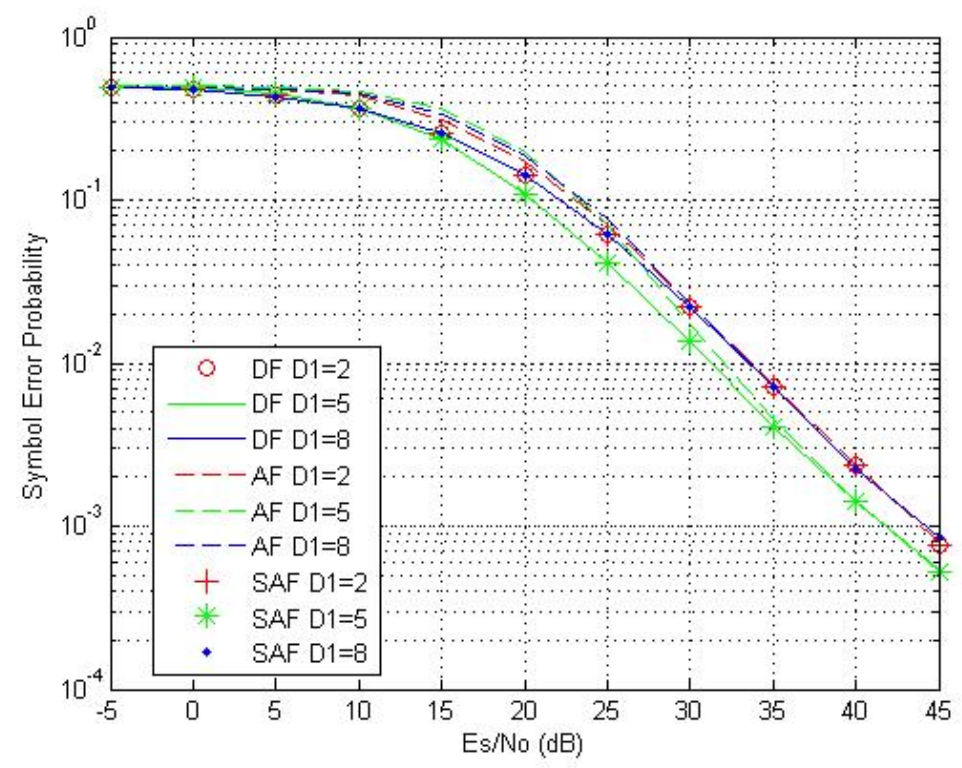

Figure 10. Symbol error performance for different distance allocations

Figure 10 shows performance for different locations of relay. In the higher $E_{s} / N_{0}$ range, placing the relay in the middle of source and destination generates best performance for all three schemes. In the lower $E_{S} / N_{0}$ range, the location of relay has little effect on the performance. In addition, the three schemes perform identically in $E_{s} / N_{0}$ regime larger than $35 \mathrm{~dB}$. However, in the lower $E_{s} / N_{0}$ range, SAF performs the same as DF but better than AF. 


\section{CHAPTER FOUR}

\section{THE EFFECT OF MULTI-ANTENNA ON THE PERFORMANCE OF MULTI-HOP RELAY NETWORKS}

The appearance of multiple antennae system provides a great opportunity for wireless communications. Multiple antennas system is also called multi-input multi-output (MIMO). Numerous studies have demonstrated the MIMO system can considerably improve wireless communications performance without consuming additional bandwidth from different points of views [27][32-34].

The basic idea behind MIMO scheme is to transmit and receive signals by using multiple antennae. Two fundamental advantages of multiple antennae are diversity and multiplexing gain in a point-to-point communication (see Chapter Two). Furthermore, space-time codes are designed to achieve these gains simultaneously. In this chapter, we explore spatial multiplexing gain and diversity gain of MIMO in a multi-hop relay network.

\subsection{System Model of Multi-hop Networks with Multi-antenna Relays}

We consider a cooperative multi-relays network with a single source node $S$, a single destination node $D$, and multiple relay nodes. Each node carries multiple antennae. Assume the $M$ relay nodes are placed in cascading order. We continue considering that the time is slotted. In each slot, source or only one of the relays is active as shown in Figure 11. Accordingly, the communication 
process can be simplified as, at any time slot, only one node transmitting and only the subsequent node receiving. The model is depicted in Figure 11.

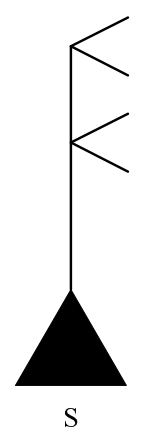

Source

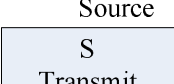

Transmit

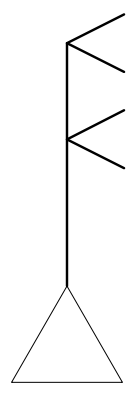

$\mathrm{R}_{1}$

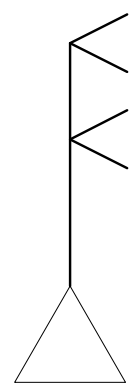

$\mathrm{R}_{2}$

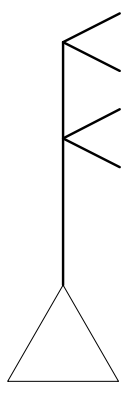

$\mathrm{R}_{\mathrm{M}}$

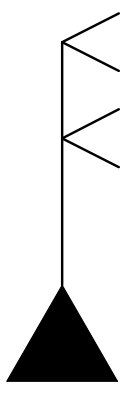

D

Destination

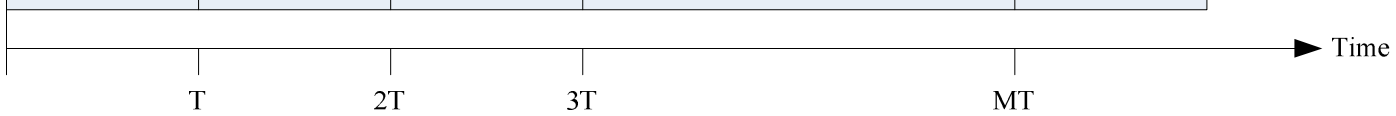

Figure 11. A cooperative relay network with multiple antennae

Compared with single-antenna system, MIMO scheme can utilize multiple channels between a transmitter and a receiver, while single-antenna system only has one channel to use.

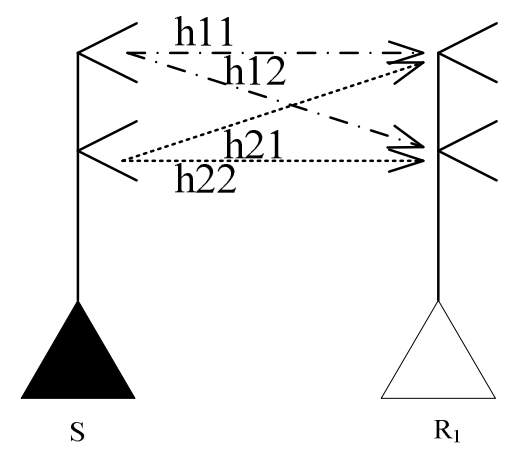

Figure 12. 2X2 MIMO channel coefficients

Take $2 \times 2 \mathrm{MIMO}$ system as example, as Figure 12 shown, the received information can be written as 


$$
\begin{aligned}
& y_{1}=h_{11} x_{1}+h_{21} x_{2}+n_{1} \\
& y_{2}=h_{12} x_{1}+h_{22} x_{2}+n_{2}
\end{aligned}
$$

Equation 11

in which $y_{1}$ and $y_{2}$ stand for the received signals from antenna 1 and antenna 2 respectively at the receiver side. $x_{1}$ and $x_{2}$ are the transmitted signals from antenna 1 and antenna 2 respectibely at the transmitter side. $n_{1}$ and $n_{2}$ are the AWGN with mean 0 and variance $\sigma^{2}$ at the receiver side.

Equation 11 can be combined as

$$
Y=\left[\begin{array}{l}
y_{1} \\
y_{2}
\end{array}\right]=\left[\begin{array}{ll}
h_{11} & h_{21} \\
h_{12} & h_{22}
\end{array}\right]\left[\begin{array}{l}
x_{1} \\
x_{2}
\end{array}\right]+\left[\begin{array}{l}
n_{1} \\
n_{2}
\end{array}\right]
$$

Let matrix $H=\left[\begin{array}{ll}h_{11} & h_{21} \\ h_{12} & h_{22}\end{array}\right], X=\left[\begin{array}{l}x_{1} \\ x_{2}\end{array}\right]$, and $N=\left[\begin{array}{l}n_{1} \\ n_{2}\end{array}\right]$. Therefore Equation 12 can be generalized to

$$
Y_{m \times 1}=H_{m \times n} X_{n \times 1}+N_{m \times 1}
$$

in which $m$ and $n$ represents the number of receiver antennas and transmitter antennas respectively. Then $H_{m \times n}$ means a wireless channel matrix with size $m \times n$. Similarly, $X_{n \times 1}$ means matrix of the transmitted signals of with size $n \times 1$, and $N_{m \times 1}$ is the noise matrix at the receiver side. $Y_{m \times 1}$ is the matrix of received signals with size $m \times 1$.

\subsection{Multi-antenna: Spatial Multiplexing}

In this subsection, we explore a simple spatial multiplexing technique, by sending $n$ independent data stream through $n$ antennas and using zero-forcing at 
the receiver. There are other spatial multiplexing techniques such as V-BLAST and D-BLAST. They are out of scope of this thesis.

In order to obtain spatial multiplexing gains $G,\left(G_{\max }=\min _{m, n}(m, n)\right)$, each antenna should transmit an independent data stream. In this case, each element of $X_{n \times 1}$ belongs to an independent data stream. Regarding the decoding of the received signals $Y$, we need to figure out a method to get rid of matrix $H_{m \times n}$. Here we adopt the linear zero-forcing algorithm that is a straightforward method to recover transmitted matrix. The algorithm can be defined as trying to find out a matrix $H_{n \times m}^{+}$to satisfy that $H_{n \times m}^{+} H_{m \times n}=I_{n \times n}$ in which $H_{n \times m}^{+}$is the famous Moore-Penrose pseudo inverse of $H_{m \times n} . H_{n \times m}^{+}$is defined as

$$
H_{n \times m}^{+}=\left(H_{n \times m}^{c o n j} H_{m \times n}\right)^{-1} H_{n \times m}^{c o n j}
$$

where $H_{n \times m}^{c o n j}$ is the conjugate transpose of $H_{m \times n}$ and $(\cdot)^{-1}$ is the inverse operator.

In the thesis, we consider that the number of antennas at the transmitter side is the same as the number of antennas at the receiver side. For the case, zero-forcing algorithm can be simplified to $H^{-1} H=I$, in which $H^{-1}$ is the reverse of $H$. In the following, In the following we use $H_{n \times n}^{-1}$ to multiply the received signals matrix, we achieve

$$
H_{m \times n}^{-1} Y_{m \times 1}=H_{m \times n}^{-1} H_{m \times n} X_{n \times 1}+H_{m \times n}^{-1} N_{m \times 1} \quad \text { Equation } 15
$$

where $m=n$. Then the transmitted signals $X_{n \times 1}$ can be obtained as

$$
H_{m \times n}^{-1} Y_{m \times 1}=X_{n \times 1}+H_{m \times n}^{-1} N_{m \times 1} \quad \text { Equation } 16
$$


In summary, the decoding process can be considered as using reverse of the channel matrix to multiply by the received signals $Y$.

In the model, we keep using the simplified path-loss model as described in Section 3.1. After adding the path-loss model, based on Equation 1, the channel matrix $H$ is modified to $\bar{H}=H \sqrt{1 / d^{\alpha}}$, where $d$ is the distance between the transmitter and the receiver.

As for the relay strategies, we continue employing the two famous schemes, amplify-and-forward and decode-and-forward, as well as the saturated amplify-and-forward scheme we proposed, referring to Section 3.2.

Take $m=2$ and $n=2$ for example. The source divides transmitted signals to two groups, obeying the law that if the symbol is assigned to one antenna then the next symbol is assigned to another antenna. At the receiver side, the destination needs to combine all received signals from the two antennas following the same rule, as shown in Figure 13.

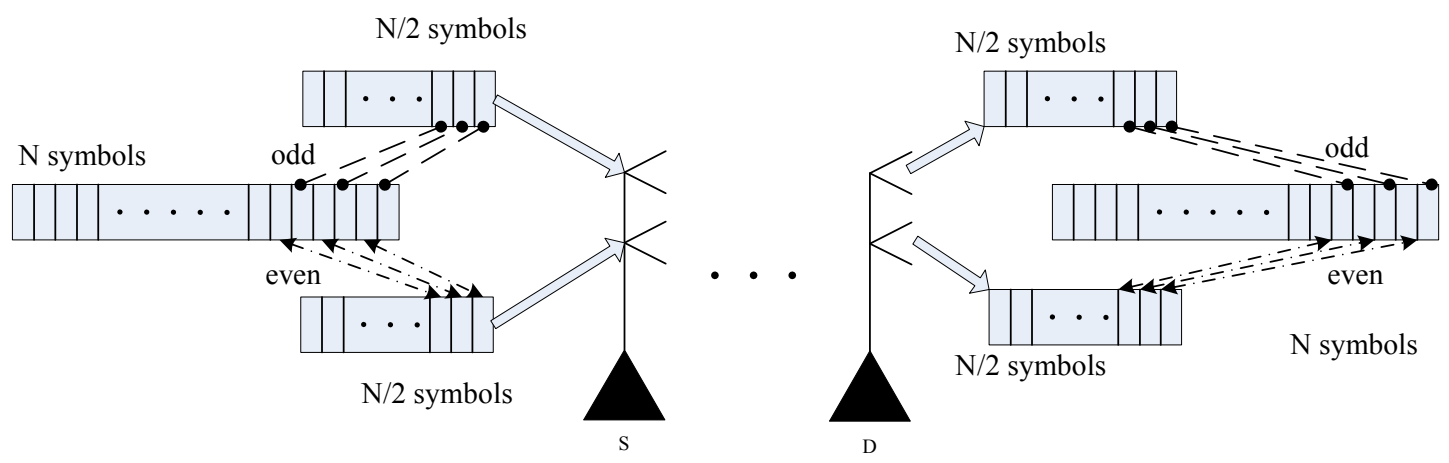

Figure 13. The rule of division and combination signals for $2 \mathrm{X} 2 \mathrm{MIMO}$ 
Figure 13 demonstrates the rules for signals division at the source and signals combination at the destination based on the $2 X 2 \mathrm{MIMO}$. At the transmitter side, it assigns the transmitted signals that are at odd positions to one antenna and the transmitted signals that at even positions to another antenna. At the receiver side, it places received signals from one antenna to odd positions of the final combined signals and the ones from another antenna to even positions of the final combined signals.

Furthermore, from Figure 13, it is obvious to conclude that transmitting rate is doubled by two antennas. However, it is hard to comment on the diversity gain. Therefore we set up a simulation to verify whether the diversity gain is obtained or not. We consider a multi-hop network based on the model in Section 4.1. There are $M=4$ relays. BPSK modulation is adopted and nodes transmit with equal power. The distance between source and destination is $10 \mathrm{~m}$ and path-loss exponent is $\alpha=2.5$. The transmitted signals are divided following the rule as shown in Figure 13, and the received signals are combined following the same rule. In addition, a single-antenna multi-hop network is considered as well. Based on the same specifications, the symbol error performances are obtained in Figure 14. 


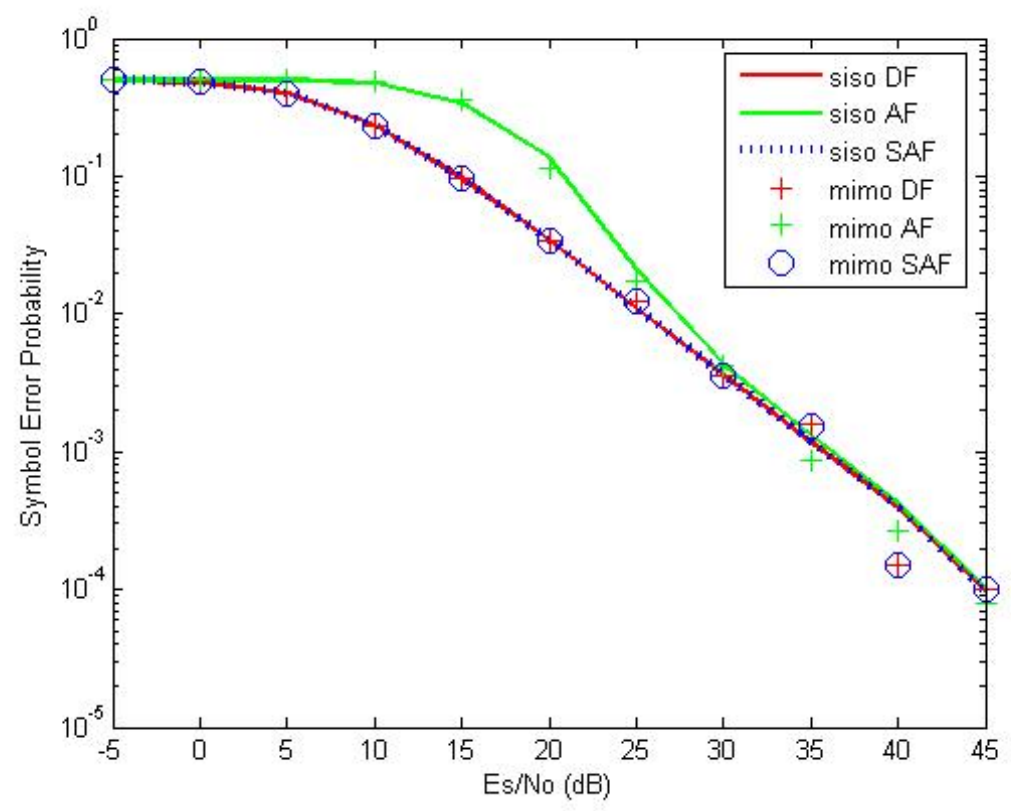

Figure 14. Symbol error performance comparison between MIMO and SISO

Figure 14 compares symbol error rate of multi-antenna relay network and single-antenna relay network. Obviously, in this model using multiple antennae does not improve the symbol error rate performance compared with using a single antenna. Hence, diversity gain is not obtained.

\subsection{Multi-antenna: Diversity Gain}

In this subsection, we explore a simple diversity technique, by sending the same data stream through $n$ transmit antennas and using zero-forcing at the receiver (see Section 4.2). In this case, we can rewrite Equation 13 as

$$
Y_{m \times 1}=H_{m \times n} 1_{n \times 1} X_{1 \times 1}+N_{m \times 1}
$$


where $1_{n \times 1}$ denotes a vector of ones with size $n$ by 1 . Obviously, Equation 17 can be simplified to

$$
Y_{m \times 1}=H_{m \times 1} X_{1 \times 1}+N_{m \times 1}
$$

Since $H_{m \times 1}$ is a vector of sum of $n$ channel coefficients, the zero-forcing receiving simply becomes multiplying $Y$ with $\frac{h_{i}{ }^{H}}{\left|h_{i}\right|}$, where $h_{i}$ is $i^{\prime}$ th element of $H_{m \times 1}$ and $h_{i}{ }^{H}$ is the conjugate transpose of $h_{i}$.

In order to verify the space diversity is obtained through this technique, we adopt the same model used in Section 4.2 through which Figure 14 has been generated. But we send same data streams through 2 antennas.

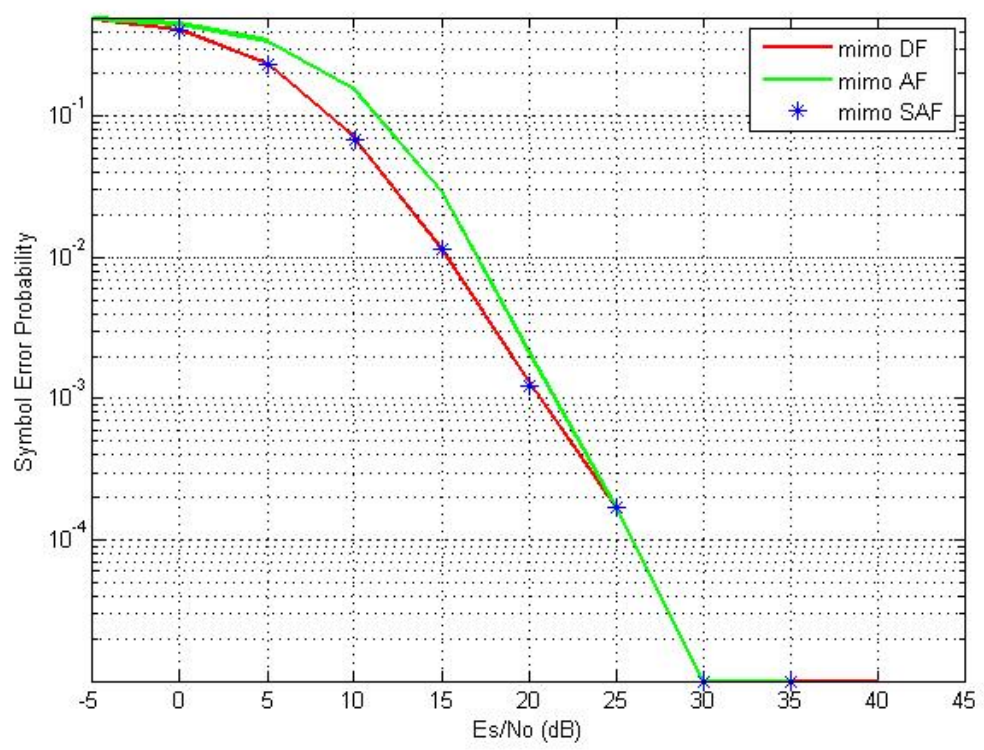

Figure 15. Symbol error performance of MIMO to verify space diversity Compared with Figure 14, Figure 15 demonstrates that symbol error rate decreases much faster with $E_{S} / N_{o}$ than it does in Figure 14, especially in high 
$E_{s} / N_{o}$ region. It has been well known that for a single-antenna Rayleigh fading channel, the average error probability decays with $1 / S N R$ in high $E_{S} / N_{o}$ regime [27], which has been shown in Figure 14. For an independent multi-antenna Rayleigh fading channel, if diversity gain is $n$, the average error probability decays with $1 / S N R^{n}$ at high SNR region [27]. From Figure 15, it is reasonable to estimate $n=2$. Thus, we can conclude that transmitting same data through two antennas can obtain diversity gain of 2 . 


\section{CHAPTER FIVE}

\section{COOPERATIVE SIGNALING FOR MULTI-STAGE RELAY NETWORK}

\subsection{Motivation of Multi-stage Relay Networks}

MIMO system is capable of either improving communication performance through spatial diversity or increasing the transmitting data rate through spatial multiplexing. Nowadays plenty of researches have been performed to learn how to take both advantages [3-6]. However, multiple antennae are not attractive in practical applications such as wireless sensor network, ad-hoc networks, and cellular networks because in these networks communication nodes are always limited in size, cost, and hardware complexity. Therefore, in order to apply benefits of MIMO system to these networks that are not able to support multiple antennae, a new form of diversity, called cooperation diversity, was proposed based on the cellular scenario in [8]. Besides cellular networks, wireless ad-hoc network also have the potential to implement cooperation diversity successfully, which was discussed in [35].

Basically, cooperative diversity can be achieved by the collaboration of a group of relays with each relay owning a single antenna. When one relay tries to transmit signals, the neighbors who are idle can share their antennas to transmit signals at the same time. In the transmission, the objective relay and the neighbors who provide assistance are forming a virtual MIMO system even though they each have only one antenna. 
In this chapter, we are going to introduce a network formed by multiple stages with each stage consisting of multiple relay nodes. Each node carries only one antenna and each node cooperates with each other when doing transmitting and receiving, which can generate special diversity as MIMO does. We adopt distributed space-time coding (DSTC) to extract spatial diversity gain in addition to multiplexing gain, which has been proven in $[9,13,36]$.

Unfortunately, most discussions and analyses of cooperative diversity protocols are developed under high signal-to-noise (SNR) regime [9]. However, it is not achievable by most wireless sensor networks or ad-hoc networks in which the relay nodes are limited in battery power. In addition, the most commonly proposed selection relaying schemes cannot satisfy these applications either. Selection relaying schemes permit only the relays that are able to decode the source signals correctly to retransmit [14]. In low-to-moderate SNR environments, the relay nodes that can decode the received signals perfectly are in minority, or even worse no one can make it. In this case, the communication may be stopped at the relay nodes. As a result, it is not wise to perform the selection relay strategy in low-to-moderate SNR environments.

A new scheme has been proposed in [24]. It highlights developing and analyzing cooperative schemes for wireless networks that operate at a low-tomoderate SNR regime. The scheme utilizes a suitable preprocessing function to process the received signals and then use DSTCs to encode the treated signals before retransmitting. By the new scheme, the relays that decode the received 
signals unsuccessfully will not be neglected. Instead they will go through appropriate preprocessing and will retransmit. Accordingly, the scheme is capable of taking advantage of as many relays as possible to cooperate. However, the current work is focusing only on a single stage relay network [24]. No path-loss propagations are involved. No Rayleigh fading is considered. These assumptions are far from practical applications. Therefore, in this work we extend the network to a multi-stage relay network with a flat Rayleigh fading channel. A path-loss model is considered among all propagations from the source to relays, relays to relays, and relays to the destination.

\subsection{System Model of Multi-stage Relay Networks}

There are several premises in this work. The first promise is that symbolby-symbol encoding and decoding are considered. Therefore no packet transmissions are involved. Second, we assume a zero channel estimation error at the receiver side.

The system model we adopt is depicted in Figure 16. We consider a relay network consisting of a single source $S$, a single destination $D$, and $L$ relay stages with $K$ relay nodes in each stage. Each relay holds one antenna, and the $K$ relay nodes in one stage cooperate with each other to form a virtual multiple antennae system. We consider a two-phase communication: one is the source broadcasting signals, and the second is relay retransmitting. Specifically, the transmission process contains $L+1$ time slots. In the first time slot, the source 
broadcasts signals, and the first relay stage receives the signals. In the second time slot, the first relay stage preprocesses the received signals and encodes them, and finally retransmits them. Similarly in the following time slots, the rest relay stages will perform the same process. Finally, in the last time slot destination receives signals from the last relay stage and decodes them. We assume that relays utilize only the received signals from the previous relay stage.

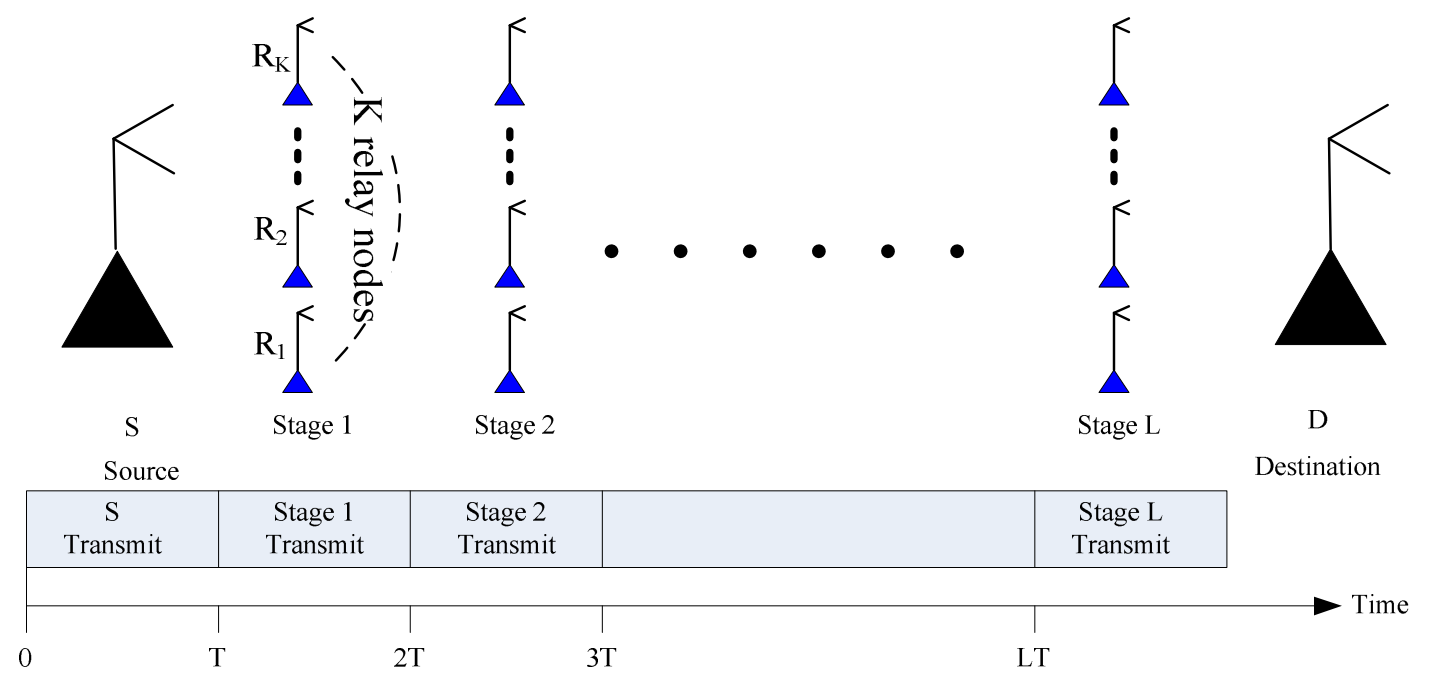

Figure 16. A Cooperative relay network with multiple stages

Compared with the model we employ in Section 3.1, the model extend a single relay node to multiple relay nodes in each stage. Let the source $S$ transmit signals $\left[s_{1} \cdots s_{N}\right]^{T}$ with average power $P_{S}$ that satisfies $P_{S}=E\left\{|\bar{s}|^{2}\right\} . E\{\cdot\}$ means the expectation operator and $N$ is the total number of symbols transmitted by the source. Let $\bar{r}_{l}^{[j]}=\left[r_{i 1}{ }^{[j]} \cdots r_{i N}{ }^{[j]}\right]^{T}$ denote the received signals at the $i^{\prime}$ th relay in the $j^{\prime}$ th stage. Therefore, the received signals of the $i$ 'th relay in the first stage can be written as 


$$
\bar{r}_{l}^{[1]}=h_{i}^{[1]} \bar{S}+{\overline{W_{l}}}^{[1]}
$$

Equation 19

where ${\overline{w_{l}}}^{[1]} \in{\overline{w_{l}}}^{[j]}=\left[w_{i 1}{ }^{[j]} \cdots w_{i N}{ }^{[j]}\right]^{T}$ denotes the zero-mean complex Gaussian random noise with variance $\sigma_{i}^{2}$ in the $j^{\prime}$ th stage. $h_{i}{ }^{[1]}$ represents the flat Rayleigh fading channel coefficients between source and the $i^{\prime}$ th relay in the first stage.

In the work to make the path-loss model more practical, we modify the path-loss model in Section 3.1 slightly to

$$
\sigma_{i}^{2} \begin{cases}\propto \frac{1}{d_{i}^{\alpha}} & d_{i} \geq 1 \\ =1 & d_{i}<1\end{cases}
$$

where $\sigma_{i}^{2}$ is the variance of the path-loss power in the $i$ 'th link between stage $T_{j-1}$ and $T_{j} . d_{i}$ is the distance of the $i$ 'th link. $\alpha$ is the path-loss exponent.

5.3 Relay Processing: Preprocessing, Mapping, and DSTCs

\subsubsection{Relay Processing in the First Stage}

After the relays in the first relay stage receive the signals from the source, the relays preprocess the received signals and apply DSTCs afterward. The relay processing in a single relay node can be summarized in Figure 17. 


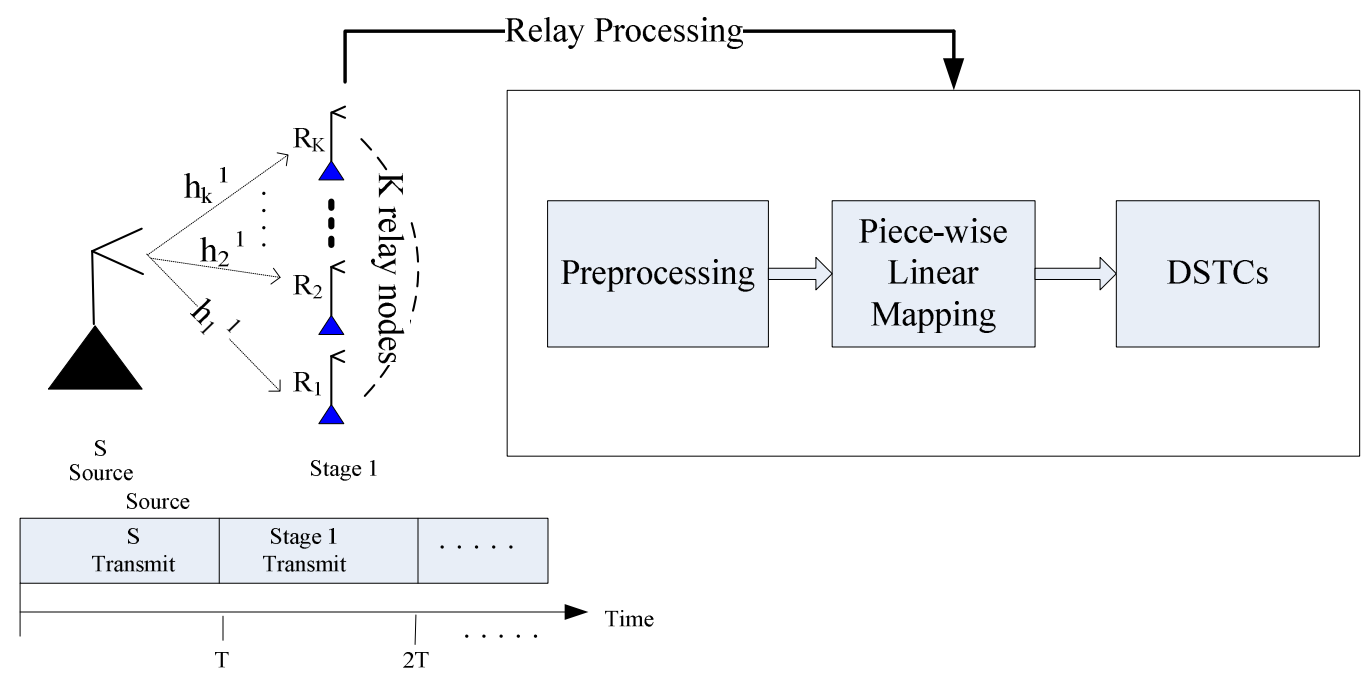

Figure 17. Processing of each relay node in multi-stage relay network

Figure 17 highlights the relay processing that includes three phases, preprocessing, piece-wise linear mapping, and DSTCs. In the following, the relay processing will be discussed in detail based on the source and the first stage link.

Let $\bar{u}_{l}^{[j]}=\left[u_{i 1}{ }^{[j]} \cdots u_{i N}{ }^{[j]}\right]^{T}$ denote the signals after preprocessing at the $i$ 'th relay of the $j$ 'th stage. Then at the first stage, the preprocessed signals can be written as

$$
\bar{u}_{l}^{[1]}=\frac{h_{i}^{[1]^{H}}}{\left|h_{i}{ }^{[1]}\right|^{2}} \bar{r}_{l}^{[1]}
$$

where $h_{i}{ }^{[1]^{H}}$ denotes the conjugate transpose of $h_{i}^{[1]}$.

In the process of piece-wise linear mapping, a generalization function $F(\cdot)$ is applied, as discussed in Section 3.2. After using piece-wise linear mapping function to process the preprocessed signals, we gain retransmitted signals 
$\bar{t}_{l}^{[1]} \in \bar{t}_{l}^{[j]}=\left[t_{i 1}{ }^{[j]} \cdots t_{i N}{ }^{[j]}\right]^{T}$ at the $i^{\prime}$ th relay of the first stage as

$$
\bar{t}_{l}^{[1]}=F\left(\bar{u}_{l}^{[1]}\right)
$$

Equation 22

Define that

$$
F\left(\bar{u}_{\imath}^{1}\right)=F\left(R E\left(\bar{u}_{\imath}^{1}\right)\right)+j * F\left(\operatorname{IM}\left(\bar{u}_{\imath}^{1}\right)\right)
$$

where $R E(\cdot)$ and $I M(\cdot)$ means real and imaginary part operator respectively. $F(\cdot)$ is a represents the piece-wise linear mapping function. Then we can perform mapping on real part and imaginary part respectively such that

$$
\begin{aligned}
& R E\left(\bar{t}_{l}^{1}\right)=F\left(\operatorname{RE}\left(\bar{u}_{\imath}^{1}\right)\right) \\
& \operatorname{IM}\left(\bar{t}_{l}^{1}\right)=F\left(\operatorname{IM}\left(\bar{u}_{l}^{1}\right)\right)
\end{aligned}
$$

Next, we will focus on DSTCs. It is well known that Alamouti code are the simplest one among all space-time codes, therefore we apply it to our work. At each stage there are two relay nodes for retransmitting and receiving. Let the transmitted signals be $\bar{t}_{1}^{[1]}$ and $\bar{t}_{2}^{[1]}$ in the first stage at relay 1 and relay 2 , respectively. For simplicity, set $\left.\bar{t}_{1}^{[1]}=\left[\begin{array}{ll}t_{11}{ }^{[1]} & t_{12}\end{array}\right]^{[1]}\right]^{T}$ and

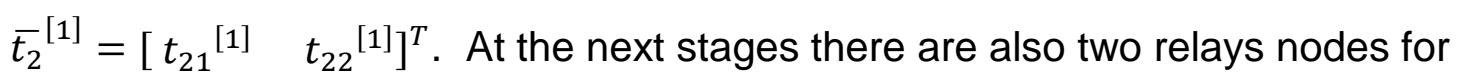
receiving. Then ${\overline{r_{1}}}^{[2]}$ and ${\overline{r_{2}}}^{[2]}$ denote the received signals at the first relay node and the second relay node respectively in the second stage. After Alamouti coding, $\bar{r}_{1}^{[2]}$ can be written as

$$
\bar{r}_{1}^{[2]}=\left[\begin{array}{ll}
h_{11}^{[2]} & h_{12}^{[2]}
\end{array}\right]\left[\begin{array}{cc}
t_{11}^{[1]} & -t_{12}^{[1]^{*}} \\
t_{22}^{[1]} & t_{21}^{[1]}
\end{array}\right]+\bar{n}_{1}^{[2]}
$$


And ${\overline{r_{2}}}^{[2]}$ can be written as

$$
\bar{r}_{2}^{[2]}=\left[\begin{array}{ll}
h_{21}^{[2]} & h_{22}^{[2]}
\end{array}\right]\left[\begin{array}{cc}
t_{11}^{[2]} & -t_{12}^{[2]^{*}} \\
t_{22}^{[2]} & t_{21}^{[2]^{*}}
\end{array}\right]+\bar{n}_{2}^{[2]}
$$

where $h_{11}^{[2]}, h_{12}^{[2]}, h_{21}^{[2]}$, and $h_{22}^{[2]}$ denote the four independent flat fading Rayleigh channel coefficients between stage 1 and stage 2 respectively. $\bar{n}_{1}^{[2]}$ and $\bar{n}_{2}^{[2]}$ are two independent noise vectors of relay 1 and relay 2 respectively in the second stage. These vectors are presented in Figure 18.

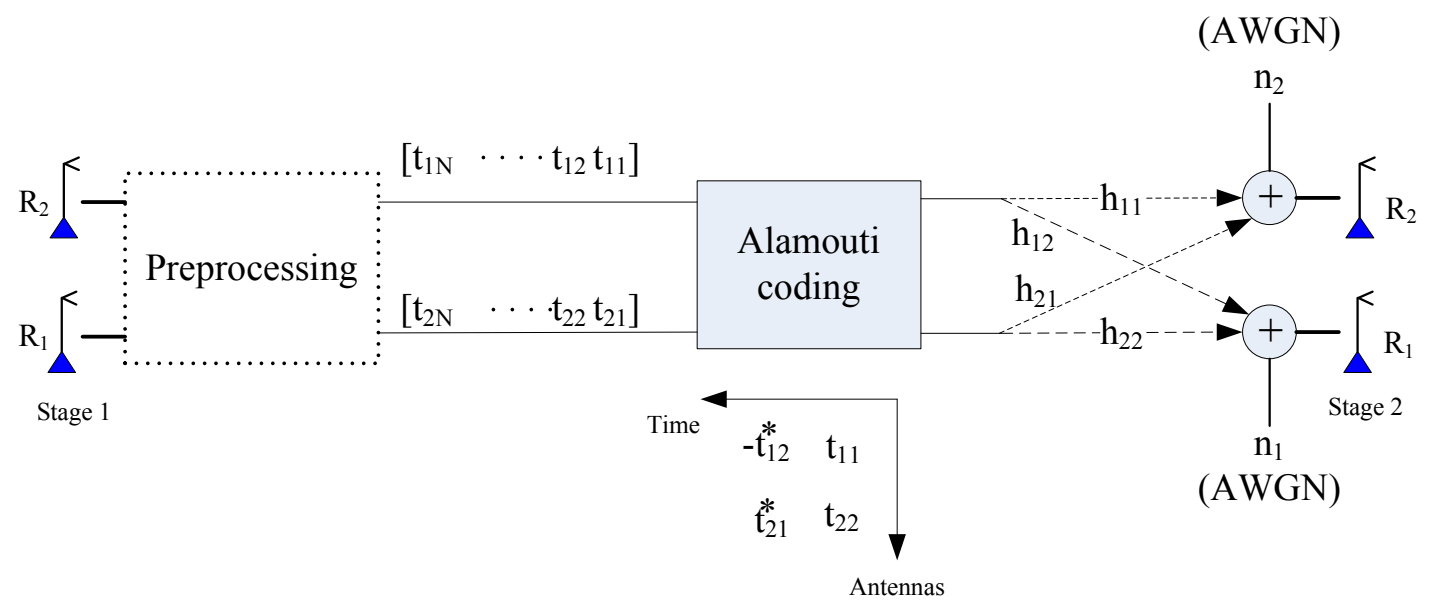

Figure 18. Alamouti coding

Figure 18 shows that $h_{11}^{[2]}$ and $h_{11}^{[2]}$ represent the channel coefficients from relay 1 and relays 2 in the first stage to the relay 1 in the second stage respectively. Similarly, $h_{21}^{[2]}$ and $h_{22}^{[2]}$ represent the channel coefficients from relay 1 and relays 2 in the first stage to the relay 2 in the second stage respectively. $\bar{n}_{1}^{[2]}=\left[\begin{array}{ll}n_{11}^{[2]} & n_{12}^{[2]}\end{array}\right]$ is the zero-mean AWGN vector with variance $\sigma_{1}^{2}$ of relay 1 in stage 2 , in which $n_{11}^{[2]}$ is the noise for the first time slot and $n_{12}^{[2]}$ is the noise for the 
second time slot. Likewise, $\bar{n}_{2}^{[2]}=\left[\begin{array}{ll}n_{21}^{[2]} & n_{22}^{[2]}\end{array}\right]$ represents the same information of relay 2 in stage 2 .

Except for preprocessing, mapping and Alamouti encoding for the first stage can be easily generalized to following stages. Generally orthogonal spacetime codes can be used if the number of nodes in each stage is large than 2 .

\subsubsection{Preprocessing in Following Stages}

Preprocessing in the second stage and the following stages is different from the one we described above because the transmission models are different. In the first stage, there is only one node transmitting, while in the following stages there are more than one nodes transmitting, as Figure 18 shown. In this case, we cannot easily use the Equation 21 because channel coefficients have been extended to a complex matrix. In addition, Alamouti encoding is involved. Therefore, standard Alamouti decoding is considered when we discuss the preprocessing of the second stage and following stages. Here we take the second stage as an example and set $K=2$ for simplicity.

Following Equation 25 and Equation 26, the received signals, $\bar{r}_{1}^{[2]}$ and $\bar{r}_{2}^{[2]}$ at relay 1 and relay 2 can be calculated respectively. Basically two relay nodes perform same processes, so we just analyze one relay node as reference. Then $\bar{r}_{1}^{[2]}$ can be given in matrix notation as follows

$$
\left[\begin{array}{l}
r_{11}^{2} \\
r_{12}^{2}
\end{array}\right]=\left[\begin{array}{ll}
h_{11}^{2} & h_{12}^{2}
\end{array}\right]\left[\begin{array}{cc}
t_{11}^{1} & -t_{12}^{1 *} \\
t_{22}^{1} & t_{21}^{*}
\end{array}\right]+\left[\begin{array}{ll}
n_{11}^{2} & n_{12}^{2}
\end{array}\right]
$$

It can be also written as 


$$
\left[\begin{array}{c}
r_{11}^{2} \\
r_{12}^{2}
\end{array}\right]=\left[\begin{array}{cc}
h_{11}^{2} & h_{12}^{2} \\
h_{12}^{2} & -h_{11}^{2}
\end{array}\right]\left[\begin{array}{l}
t_{11}^{1} \\
t_{22}^{1}
\end{array}\right]+\left[\begin{array}{c}
n_{11}^{2} \\
n_{12}^{2}
\end{array}\right]
$$

Let $H=\left[\begin{array}{cc}h_{11}^{2} & h_{12}^{2} \\ h_{12}^{2}{ }^{*} & -h_{11}^{2}\end{array}\right]$. In order to obtain meaningful signals $\left[\begin{array}{l}t_{11}^{1} \\ t_{22}^{1}\end{array}\right]$, we need to eliminate matrix $H$. As discussed in Section 3.1, use inverse of $H$ to multiply $\left[\begin{array}{c}r_{11}^{2} \\ r_{12}^{2}\end{array}\right]$ and obtain

$$
H^{-1}\left[\begin{array}{c}
r_{11}^{2} \\
r_{12}^{2 *}
\end{array}\right]=H^{-1} H\left[\begin{array}{l}
t_{11}^{1} \\
t_{22}^{1}
\end{array}\right]+H^{-1}\left[\begin{array}{c}
n_{11}^{2} \\
n_{12}^{2 *}
\end{array}\right]
$$

where $H^{-1}$ is the inverse of $H$ and $H^{-1} H=I$. Therefore Equation 29 can be simplified as

$$
\left[\begin{array}{l}
t_{11}^{1} \\
t_{22}^{1}
\end{array}\right]=H^{-1}\left[\begin{array}{c}
r_{11}^{2} \\
r_{12}^{2}
\end{array}\right]-H^{-1}\left[\begin{array}{c}
n_{11}^{2} \\
n_{12}^{2}
\end{array}\right]
$$

Equation 30 demonstrates the results of preprocessing. The results will go through following mapping and DSTCs. Generalization the second stage to the $j$ 'th stage is clearly possible.

\subsubsection{Optimal Maximum Likelihood Decoding}

The objective in this study is to find the best piece-wise linear mapping function $F(\cdot)$ so that the system average symbol error rate is minimized under the given detector (or optimal maximum likelihood detector). In mathematical term, find $\ddot{F}$

$$
\ddot{F}=\arg \min _{F} P_{e}(F)
$$


where $P_{e}(F)=P\left(\tilde{s}_{i} \neq 1 \mid s_{i}=1\right) . \quad \tilde{s}_{i}$ and $s_{i}$ denote $i$ 'th estimated symbol at the destination and the corresponding transmitted symbol at the source respectively based on the assumption that only two relay nodes are involved in each stage.

The optimal maximum likelihood (ML) decoder needs to estimate two transmitted symbols $\left(\begin{array}{rr}\tilde{s}_{i} & \tilde{s}_{i+1}\end{array}\right)$ jointly

$$
\left\{\tilde{s}_{i}, \tilde{s}_{i+1}\right\}=\arg \max _{\left\{\tilde{s}_{i}, \tilde{s}_{i+1}\right\}} p_{F}\left(\bar{r} \mid h_{11}, h_{12}, s_{i}, s_{i+1}\right) \quad \text { Equation } 32
$$

where $\bar{r}=\left[\begin{array}{c}r_{i} \\ r_{i+1}\end{array}\right]$ and $p_{F}(\bar{r} \mid \cdot)$ represents the conditional probability density function (PDF) of $\bar{r}$. From Equation 25, we can derive the probability density function of $\bar{r}$ as

$$
p_{F}\left(\bar{r} \mid h_{11}, h_{12}, s_{i}, s_{i+1}\right)=\prod_{i=1}^{2} p_{F}\left(r_{i} \mid h_{11}, h_{12}, s_{i}, s_{i+1}\right) \quad \text { Equation } 33
$$

For equally likely signals, ML decoder can be simplified to maximize $p_{F}\left(\bar{r} \mid h_{11}, h_{12}, s_{i}, s_{i+1}\right)$. Generally optimization over relay functionality is of high complexity [32][37]. The closed-form expressions are not easy to find. Numerical integration can be used for simulation purposes.

In this work, multiple relay stages are involved, which makes the implementation more complicated. Therefore, we choose standard Alamouti decoder to obtain the suboptimal performance in current simulations.

\subsection{Simulations Based on Multi-stage Relay Networks}

In this section, based on the multi-stage network model, several simulations are highlighted to compare the performance of the saturated amplify- 
and-forward (SAF) mapping function with performances of other three current well known schemes, amplify-and-forward (AF), decode-and-forward (DF), and minimum mean square error (MMSE) estimated-and-forward (EF). The performance is demonstrated by the curve of the symbol error probability as a function of symbol noise radio $E_{S} / N_{0} . E_{S}$ is the average power of transmitted signals in the source. $N_{o}$ is the variance of the AWGN noise at consecutive stages. For simplicity we assume only two relay nodes are placed in each stage. In the first stage the first relay decodes received signals perfectly and the second relay has received signals at $5.3 \mathrm{~dB}$. In the second and following stages, both relays suffer from noise with variance $N_{0}$. In addition, we use exhaustive searching method to obtain the optimal threshold $\tau$ for each stage so that the overall system performance is optimized.

We also assume that all nodes transmit with equal unit power and the distance between source and destination is set to 5 meters. The source and relays use BPSK modulation. Flat Rayleigh fading is considered and channel coefficients $\bar{h}^{i}$ in every link are independent identically distributed. The path-loss exponent is set to 2.5. All schemes are implemented respectively based on the specification.

\subsubsection{The Effect of Mapping Functions on the Performance of Single Stage} Networks

We consider a single stage relay network with relay located in 0.5 meter away from the source and obtain Figure 19. 


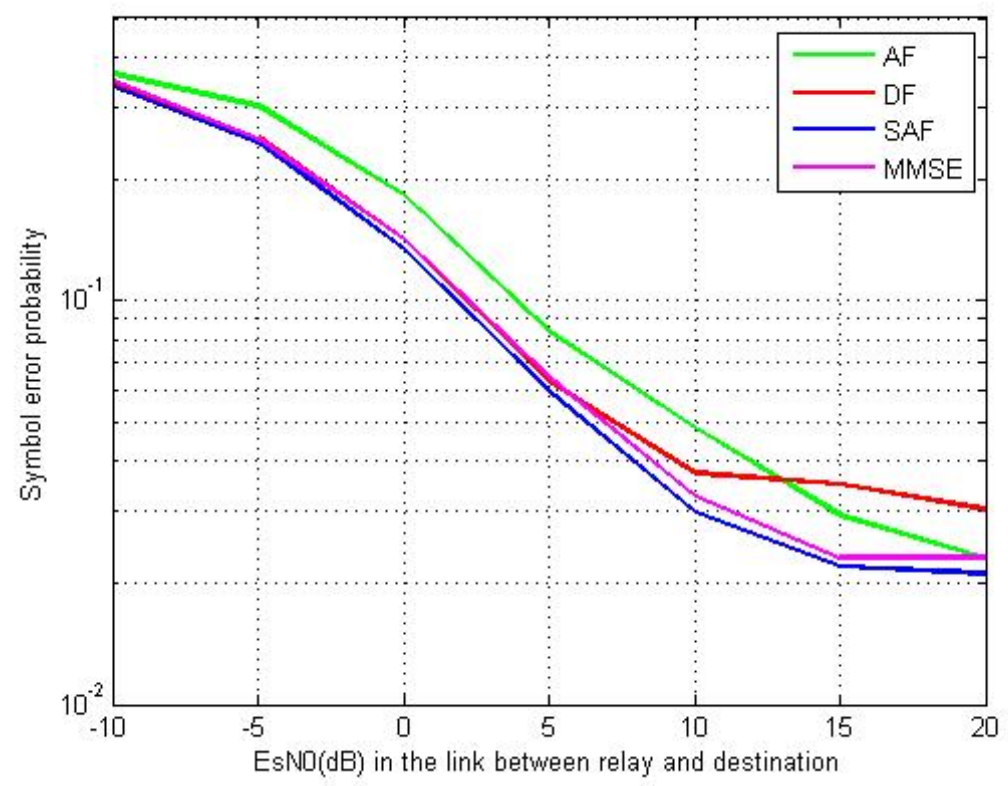

Figure 19. Comparison of mapping function in one stage relay network It is obvious to note that the saturated amplify-and-forward scheme we proposed outperforms other three schemes uniformly at the a low-to-moderate $E_{S} / N_{0}$ range. MMSE forwarding is the second optimal scheme. In the low $E_{S} / N_{0}$ range, from $-10 \mathrm{~dB}$ to $12 \mathrm{~dB}$, decode-and-forward (DF) outperforms amplify-andforward (AF), while in the higher $E_{s} / N_{0}$ range, from $12 \mathrm{~dB}$ to $20 \mathrm{~dB}$, AF performs better.

\subsubsection{The Effect of the Stage Locations on the Performance of Multi-stage} Networks

The objective of the simulation is to verify how the relay stage location plays a role in the performance of multi-stage network. We continue using the a single stage relay network model. Move the relay stage from source to 
destination at step of $0.5 \mathrm{~m}$ and plot the Figure 20 . It is worth noting that the performance is evaluated in terms of average symbol error rate versus the distance of the first link between source and the first stage curve at fixed $E_{S} / N_{0}=25 \mathrm{~dB}$.

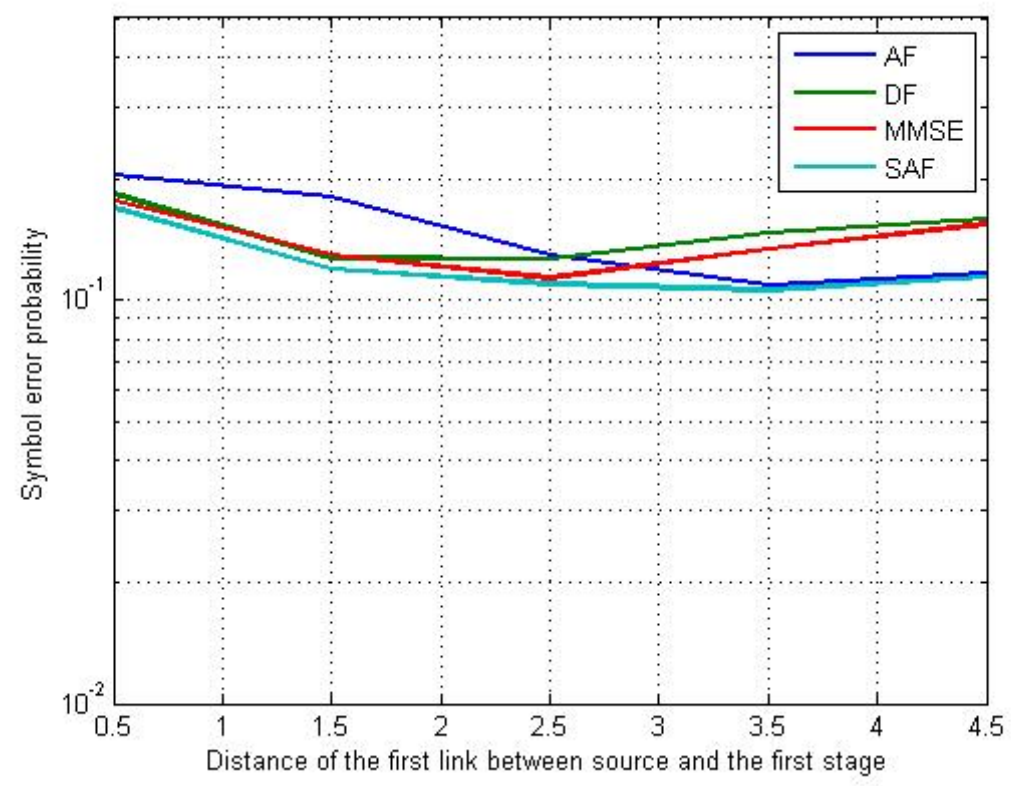

Figure 20. Performance comparison as a function of relay location

Clearly, SAF outperforms other schemes for any relay location between the source and the destination. When the relay stage is placed closer to the source, DF performs better than other two schemes. While the relay stage is placed closer to the destination, AF performs better. When the relay stage is put in the middle area of the link, MMSE performs better. 
5.4.3 The Effect of the Number of Stages on the Performance of Multi-stage Networks

We set an array of different number of relay from $L=1$ to $L=5$ respectively and plot the results in the same figure. The total distance between source and destination is 5 meter.

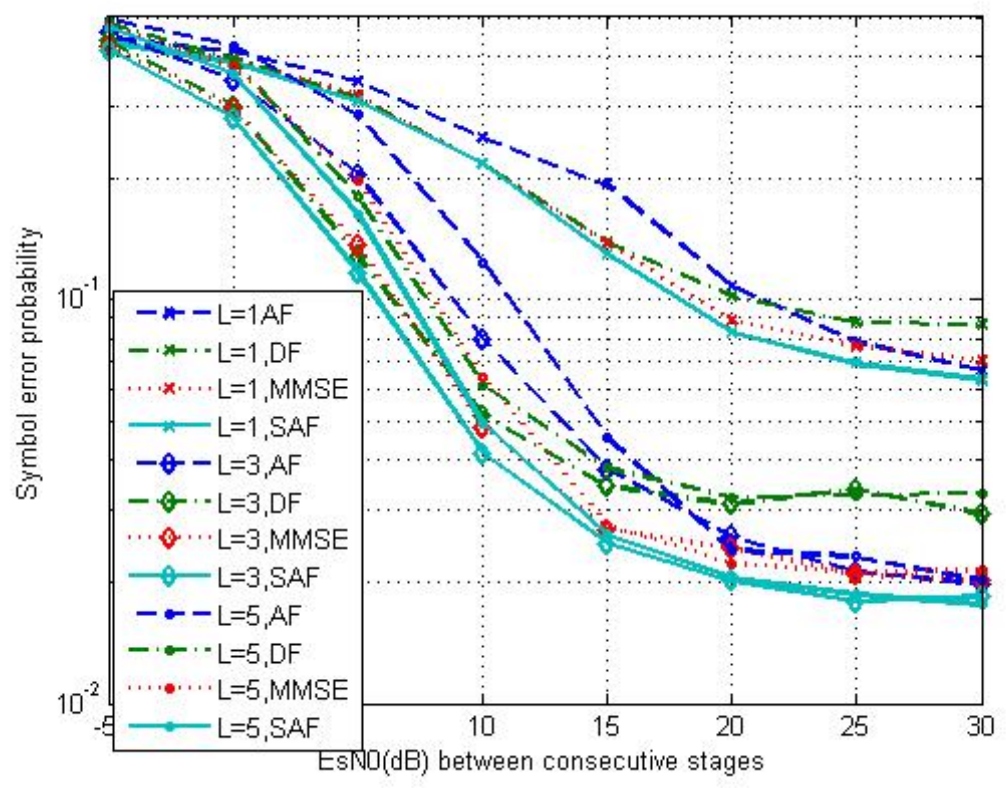

Figure 21. Comparison of mapping function in a multi-stages relay network

Figure 21 displays that for all stages, the newly proposed scheme SAF performs best among all the schemes consistently in the whole low-to-moderate $E s / N_{0}$ range. It is interesting to observe that $L=3$ is the optimal number of relay stages to be put between the destination and the source under the current assumption. And also we test other cases, such as setting distance between source and destination as 5 meter and the results presents that $L=5$ is the 
optimal number. There maybe some rules to decide the optimal number of relay stages for certain total distance, which is a good direction for our future research. 


\section{CHAPTER SIX}

\section{CONCLUSIONS AND FUTURE WORK}

This chapter summarizes the conclusions that have been obtained in this study and points out directions for future work.

\subsection{Conclusions}

Cooperative signaling has been successfully explored in this study based on several relay networks. These networks include multi-hop single-antenna relay networks, multi-hop multi-antenna relay networks, and multi-stage relay networks. Corresponding to these networks, several models have been constructed based on certain premises, such as independent and identically distributed Rayleigh fading channels and AWGN noises. In these networks, several well known relay strategies, such as $\mathrm{AF}, \mathrm{DF}$, and $\mathrm{EF}$, have been thoroughly analyzed. After generalizing the properties of these relay functions, a new scheme called saturated amplify-and-forward (SAF) was proposed, in which a controllable parameter called $\tau$ can be chosen optimally to obtain the optimal performance.

Single-antenna multi-hop networks have wide applications in vehicular networks. Simulations are performed to show the effect of the path-loss exponent, the effect of relay locations, and the effect of the number of relays on the performance of multi-hop relay networks. In single-antenna multi-hop 
networks, when the path-loss exponent is equal to 2 , the performance of symbol error rate is the best. In addition, the performance increases with the number of relays. Placing a relay in the middle of a source and a destination generates better performance than placing a relay close to a source or close to a destination, especially in higher $E_{s} / N_{0}$ range. From these simulations, there is a common point worthy of attention. It is that the three schemes, AF, DF and SAF, perform identically in high $E_{S} / N_{0}$ regimes larger than $35 \mathrm{~dB}$. However, in the lower $E_{S} / N_{0}$ range, SAF performs the same as DF but better than AF.

In multi-hop networks with multi-antenna relays, multiple antennae play an important role in either expanding the transmitting data rate by providing spatial multiplexing gain or improving the transition reliability by providing a spatial diversity gain.

In multi-stage relay networks, each stage has multiple single-antenna relay nodes, which build into a virtual multi-antenna system. For this system, distributed space-time codes (DSTCs) are introduced. As expected, DSTCs provide both diversity gain and spatial multiplexing gain to the performance. In addition, the newly proposed scheme, together with DSTCs, makes the new scheme clearly outperform DF and AF.

It is worth noting that in this study, there are several promises. The first promise is that relaying schemes follow symbol-by-symbol relaying, so there is no concept of packet in the study. Second, channel state information (CSI) is 
available at the receiver for both $\mathrm{AF}$ and DF schemes. For SAF, CSI is known at the transmitter in order to determine the optimal threshold $\tau$.

\subsection{Future Work}

In this study, standard Alamouti decoder is adopted to perform decoding. In general, standard Alamouti decoding is not optimal when piece-wise linear mapping is involved. Therefore it would be beneficial to use optimal decoder in the future work.

Furthermore, in this study all transmitting powers are assumed to be equal to unit power. The power distribution is not optimal either. Hence, this also points out an interesting direction of future work.

In Section 5.4.3, the simulation results present that $M=3$ is the optimal number of relay stages under that experiment model. This is an interesting observation. In general, system performance increases in direct proportion to the number of relays, as shown in Section 3.3.2. So it is another interesting followup work to study the optimal number of relays in multi-stage relay networks. 


\section{REFERENCES}

[1] S. M. Alamouti, "A simple transmit diversity technique for wireless communications," IEEE Journal on Select Areas in Communications, vol. 16, no. 8, pp. 1451-1458, Oct. 1998.

[2] Goldsmith, Wireless Communications. $1^{\text {st }}$ ed., Cambridge University Press, 2005, pp. 201.

[3] E. Telatar, "Capacity of multi-antenna Gaussian channels," European Transactions on Telecommunication, vol. 10, no. 6, pp. 585-595, Nov. 1999.

[4] G. J. Foschini and M. J. Gans, "On limits of wireless communications in a fading environment when using multiple antennas," Wireless Personal Communications, vol. 6, pp. 311-335, March 1998.

[5] E. Biglieri, J. Proakis, and S. Shamai (Shitz), "Fading channels: Information theoretic and communications aspects," IEEE Trans. Inform. Theory, vol. 44, pp. 2619-2692, Oct. 1998.

[6] V. Tarokh, N. Seshadri, and A. Calderbank, "Space-time codes for highdata-rate wireless communication: Performance criterion and code construction," IEEE Trans. Inform. Theory, vol. 44, pp. 744-765, Mar. 1998.

[7] Sendonaris, E. Erkip, and B. Aazhang, "Increasing uplink capacity via user cooperation diversity," in Proc. IEEE Int. Symp. Information Theory (ISIT'98), Cambridge, MA, Aug. 1998, p. 156.

[8] Sendonaris, E. Erkip, and B. Aazhang, "User cooperation diversity-part I: system description," IEEE Trans. Commun., vol. 51, no. 11, pp. 1927-1938, Nov. 2003.

[9] Sendonaris, E. Erkip, and B. Aazhang "User cooperation diversity-Part II: Implementation aspects and performance analysis," IEEE Trans. Commun., vol. 51, no. 11, pp. 1939-1948, Nov. 2003.

[10] Stefanov and E. Erkip, "Cooperative information transmission in wireless networks," in Proc. Asian-European ITW 2002, Breisach, Germany, Jun. 2002.

[11] J. N. Laneman and G. W. Wornell, "Exploiting distributed spatial diversity in wireless networks," in Proc. 38th Allerton Conf. Communication, Control, 
and Computing, Monticello, IL, Oct. 2000.

[12] J. N. Laneman and G. W. Wamell, "Energy-efficient antenna sharing and relaying for wireless networks," in Proc. IEEE Wireless Communications and Networking Conf. (WCNC'00), Chicago, IL, Sep. 2000.

[13] J. N. Laneman, D. N. C. Tse, and G. W. Wornell, "Cooperative diversity in wireless networks: efficient protocols and outage behavior," IEEE Trans. Inform. Theory, vol.50, no. 12, pp. 3062-3080, Dec. 2004.

[14] J. N. Laneman and G. W. Wornell, "Distributed space-time coded protocols for exploiting cooperative diversity in wireless networks," IEEE Trans. Inform. Theory, vol. 49, pp. 2415-2425, Sep. 2003.

[15] J. Laneman, E. Martinian, G. Wornell, J. Apostolopoulos, and J. Wee, "Comparing application- and physical-layer approaches to diversity on wireless channels," in Proc. IEEE Int. Conf. Communications (ICC'03), Anchorage, AK, 2003.

[16] E.C. van der Meulen, "Three-terminal communication channels," Adv. Appl. Probab., vol. 3, pp. 120-154, 1971.

[17] T. Cover and A. El Gamal, "Capacity theorems for the relay channel," IEEE Trans. Inf. Theory, vol. IT-25, no. 5, pp. 572-584, Sep. 1979.

[18] Y. Zhao, R. Adve, and T. J. Lim, "Improving amplify-and-forward relay networks: optimal power allocation versus selection," IEEE Trans. Wireless Commun., vol. 6, no. 8, pp. 3114-3123, Aug. 2007.

[19] Y. Chang and Y. Hua, "Diversity analysis of orthogonal space-time modulation for distributed wireless relays," in Proc. IEEE ICASSP2004, pp. 561-564, May 2004.

[20] Stefanov and E. Ekip, "Cooperative space-time coding for wireless network," IEEE Trans. Wireless Commun., vol. 53, pp.1804-1809, Nov. 2005.

[21] K. Azarian, H. El Gamal, and P. Schniter, "On the Achievable DiversityMultiplexing Tradeoff in Half-Duplex Cooperative Channels," IEEE Transactions on Information Theory, vol. 51, no. 12, pp. 4152-4172, Dec. 2005.

[22] Y. Jing and B. Hassibi, "Distributed space-time coding in wireless relay networks," IEEE Trans. Wireless Commun., vol. 5, pp. 3524-3536, Dec. 
2006.

[23] G. Scutari and S. Barbarossa, "Distributed space-time coding for regenerative relay," IEEE Transactions on Wireless Communications, vol. 4, no. 5, pp. 2387-2399, Sept. 2005.

[24] B. Sirkeci-Mergen, "Distributed space-time codes with relay preprocessing for low SNR source-relay links," $42^{\text {nd }}$ Asilomar Conf. on Signals, Systems and Computers, 2008.

[25] Goldsmith, Wireless Communications. $1^{\text {st }}$ ed., Cambridge University Press, 2005, pp. 24.

[26] Erik G. Larsson and Petre Stoica, Space-time Block Coding for Wireless Communications, Cambridge University Press, 2003, pp. 2-3.

[27] L. Zheng and D. Tse, "Diversity and multiplexing: a fundamental tradeoff in multiple-antenna channels," IEEE Trans. on Information Theory, vol. 49, no. 5, pp. 1073-1096, May 2003.

[28] J. N. Laneman, D. N. C. Tse, and G. W. Wornell, "Cooperative Diversity in Wireless Networks: Efficient Protocols and Outage Behavior," IEEE Trans. Inform. Theory, vol. 50, no. 12, pp. 3062-3080, December 2004.

[29] J.G. Proakis, Digital Communications, $3^{\text {rd }}$ ed., New York: McGraw-Hill, 1995, Chapter 14.3.

[30] K. S. Gomadam and S. A. Jafar, "Optimal relay functionality for SNR maximization in memoryless relay networks," IEEE Journal on Selected Areas in Communications, vol. 25, no. 2, pp. 390-401, Feb. 2007.

[31] G. Kramer, M. Gastpar, and P. Gupta. "Cooperative strategies and capacity theorems for relay networks," IEEE Transactions on Information Theory, vol. 51, no. 9, pp. 3037-3063, Sep. 2005.

[32] S. Cui, A. J. Goldsmith, and A. Bahai, "Energy-efficiency of MIMO and cooperative MIMO techniques in sensor networks," IEEE Journal on Selected Areas in Communications, vol. 22, no. 6, pp. 1089-1098, 2004.

[33] Z. Yun and M.F. Iskander, "MIMO capacity for realistic wireless communications environments," Proc. 2004 IEEE AP-S, vol. 2, pp. 12311234, June 2004. 
[34] J. F. Valenzuela-Valdés, M. A. García-Fernández, A. M. Martínez-González and D. A. Sánchez-Hernández, "The influence of efficiency on receive diversity and MIMO capacity for Rayleigh-fading channels," IEEE Trans. Antennas and Propagation, vol. 56, no. 5, pp. 1444-1450, May 2008.

[35] "Special Issue on Wireless Ad Hoc Networks," IEEE J. Select. Areas. Commun., vol. 17, Aug. 1999.

[36] J. N. Laneman, G. W. Wornell, and D. N. C. Tse, "An efficient protocol for realizing cooperative diversity in wireless networks," in Proc. IEEE Int. Symp. Information Theory (ISIT), Washington, DC, June 2001, pp. 294.

[37] Abou-Faysal and M. Medard, "Optimal Uncoded Regenaration for Binary Antipodal Signaling," in Proc. IEEE International Conference on Communications 2004, vol. 2, pp. 742-746. 